
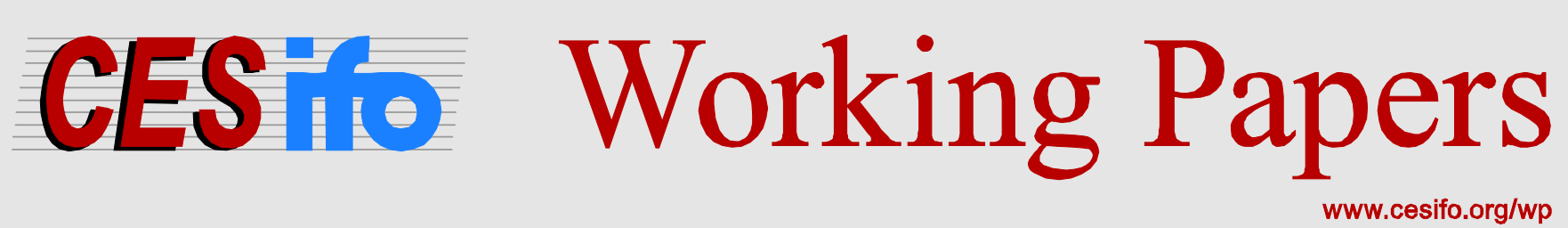

\title{
A Fairness Justification of Utilitarianism
}

\author{
Paolo G. Piacquadio
}

\author{
CESIFO WORKING PAPER NO. 5785 \\ CATEgory 2: Public CHOICE \\ FEBRUARY 2016
}

An electronic version of the paper may be downloaded

- from the SSRN website:

- from the RePEc website:

- from the CESifo website:

wWw.SSRN.com

www.RePEc.org

www.CESifo-group.org/wp 


\title{
A Fairness Justification of Utilitarianism
}

\begin{abstract}
Differences in preferences are important to explain variation in individuals' behavior. There is however no consensus on how to take these differences into account when evaluating policies. While prominent in the economic literature, the standard utilitarian criterion faces two major difficulties. First, it requires cardinal measurability and unit comparability of individuals' utilities, which cannot be inferred from individuals' observed behavior. Second, it is normatively controversial as it might support unfair policies. In this paper, we propose an alternative criterion, named opportunity-equivalent utilitarian, that overcomes these difficulties. First, our criterion ranks social alternatives on the basis of individuals' ordinal preferences, which can be estimated from individuals' observed behavior. Second, our criterion avoids the conventional critiques to utilitarianism by satisfying the following three fairness axioms: possibility of trade-offs sets a limit to the influence any individual can exert on the social ranking; non discrimination means that no individual is considered to be more deserving than any other; equal-preference transfer requires society to value positively a multicommodity progressive transfer among individuals with the same preferences. We show that, together with efficiency, continuity, and separability, these axioms force the welfare criterion to be the sum of specific indices of well-being that are cardinally measurable, interpersonally comparable, and represent each individual's preferences.
\end{abstract}

JEL-Codes: D600.

Keywords: utilitarianism, ordinal preferences, fairness, opportunities.

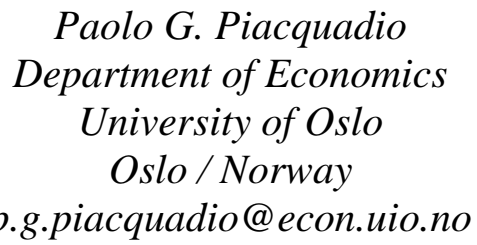

February 1, 2016 


\section{Introduction}

Differences in individuals' preferences matter. These are needed to explain individual and aggregate economic behavior and to correctly evaluate welfare effects of policies (see, among others, Kirman (1992) and, more recently, Dubois et al. (2014); Heathcote et al. (2014)). Yet, differences in preferences are problematic for welfare analysis. In the literature, there is no consensus on how to measure social welfare when individuals' have different preferences (Fleurbaey (2009)). At the same time, "confusion persists concerning the relationship between commonly used welfare indicators and well-established theoretical formulations" (Slesnick (1998), p. 2108). This led to the neglect of welfare economics by means of avoidance strategies (Atkinson (2011)): either assuming the existence of a "representative agent;" relying on a tacit agreement on the welfare criterion; or leaving the policy choice to others.

In the literature, the most prominent welfare criterion is utilitarianism, which measures social welfare by the sum of individuals' utility functions. Utilitarianism faces a major challenge in implementation: utility functions need to be unique up to increasing affine transformations (cardinally measurable) and interpersonally comparable with respect to gains and losses (unit comparable). In the view of many, however, cardinal measurability and unit comparability lack a sound empirical support (Hammond (1991)). In addition, even if this information were available, it could lead to unfair decisions (Rawls (1971); Sen (1979); Dworkin (1981)).

In this paper, we study the implications of three intuitive fairness axioms and show that these justify a class of "utilitarian" welfare criteria. These criteria are defined by the sum of indices of individuals' well-beings, which can be interpreted as utility functions. In contrast to earlier literature (d'Aspremont and Gevers (1977); Maskin (1978)), however, these indices are based on ordinal preferences only and are derived from the axioms. Since the informational basis is limited to ordinal preferences, our welfare criteria can be constructed by estimating individuals' indifference curves from observed behavior. ${ }^{1}$ Since cardinal measurability and interpersonal comparability emerge from the fairness axioms, the conventional critiques of standard utilitarianism are avoided.

\footnotetext{
${ }^{1}$ In the last decades, significant metodological improvements and the availability of detailed microlevel data makes us rather confident about economists capacity to accurately recover this information (see, among others, Heckman (2001); Halevy et al. (2015)).
} 
Beyond its simplicity, we explain the widespread use of utilitarianism in prescriptive analysis by the following equivalence. In the context of income distributions, utilitarian welfare is generally measured by:

$$
W=\sum_{i \in N} U_{i}\left(y_{i}\right)
$$

According to the standard interpretation of utilitarianism, $U_{i}$ is individual $i$ 's cardinal and unit comparable numerical representation of preferences, i.e. a function of $i$ 's income $y_{i}$. These functions $\left(U_{i}\right)_{i \in N}$ are equal across individuals as individuals are identical. They are increasing as individuals prefer more income to less. And they are concave due to the law of "decreasing marginal utility," i.e. the effect on $U_{i}$ of an additional unit of income decreases with the level of income.

A different "non-utility-based" justification can be provided. According to this view, society chooses the functions $\left(U_{i}\right)_{i \in N}$ to measure and compare individuals' wellbeings. ${ }^{2}$ These functions are equal across individuals to ensure that each individual is treated fairly: permuting their assigned incomes leaves social welfare unchanged. They are increasing to yield efficiency: if all individuals are better off, social welfare is higher. And they are concave to formalize Dalton (1920)'s "principle of transfer:" a transfer from a wealthier individual to a poorer one (leaving the first individual wealthier also after the transfer) reduces inequality and increases social welfare. Importantly, the two views are policy-equivalent, that is, $W$ represents the same social ranking, as soon as the concavity introduced due to the law of decreasing marginal utilities is the same as the concavity introduced for inequality aversion.

The extension to multicommodity spaces of standard utilitarianism is straightforward. By assumption, utility functions are cardinally measurable and unit comparable. As utility levels attained by the individuals summarize all information that is relevant for the social ranking, which bundles individuals are assigned-and, more specifically, the dimensionality of the commodity space - is irrelevant.

In this paper, we generalize the "non-utility-based" justification of utilitarianism to multicommodity spaces. Fairness principles guide social choice: society ranks allocations by evaluating individuals' assignments. Our result is that the social ranking can be expressed as the sum of specific numerical representations of individuals' preferences. Fairness principles are hereby reconciled with (i) the respect of individual preferences; and (ii) the informational requirement that social choice be based on observable behavior, that is, ordinal preferences. We introduce the following three fairness axioms.

\footnotetext{
${ }^{2}$ Among others, Atkinson (1970) defends this view for measuring income inequality in society.
} 
Possibility of trade-offs limits the influence that each individual can exert on the social ranking. To illustrate, let each individual but individual $i$ be assigned the zero bundle (by assumption the worst assignment). Then, for each individual $j \neq i$, there is a socially indifferent alternative that assigns a non-zero bundle only to $j$. In other words, the loss in well-being that individual $i$ experiences - when her bundle is reduced to zero - can be compensated by a gain in well-being of any other individual - assigning her some positive bundle instead of zero. ${ }^{3}$

Non discrimination means that no individual is considered to be more deserving than any other. ${ }^{4}$ We first illustrate the axiom in the context of income distributions. Consider a distribution which assigns income $y_{i}>0$ to individual $i$ and no income to all other individuals. Another income distribution assigns a lower income $y_{j}<y_{i}$ to individual $j$ and no income to all other individuals (including $i$ ). If society prefers the latter distribution, individual $i$ is discriminated against: society would rather assign less to $j$ than more to $i$. The axiom rules out this type of social preferences. In a multicommodity framework, society needs to account for differences in preferences. Consider all allocations at which individual $i$ is indifferent between her assignment $x_{i}$ and some bundle $\bar{x}_{i}$, while all other individuals are assigned the zero bundle. Then, $i$ is discriminated against if for each such allocation, society prefers to assign a smaller bundle $x_{j}<x_{i}$ to some individual $j \neq i$ and the zero bundle to all others (including $i$ ). ${ }^{5}$ Such discrimination is not permitted.

Equal-preference transfer introduces a social concern for inequality. This axiom is inspired by Dalton's transfer principle. Consider two individuals, $i$ and $j$, with the same preferences. ${ }^{6}$ At allocation $x$, individual $i$ is assigned a bundle on a higher indifference curve than individual $j$. At allocation $x^{\prime}$, the difference between their assignments is reduced by a transfer of commodities from $i$ to $j$, while all other individuals are unaffected. Then, society considers $x^{\prime}$ at least as desirable as $x{ }^{7}$

${ }^{3}$ This requirement is related to anonymity with respect to individuals' utilities, common in the literature (see d'Aspremont and Gevers (2002)). Anonymity is however not compelling in the present setting, in which no comparable information about preferences are available. An axiom similar to possibility of trade-offs has been introduced by d'Aspremont (1985).

${ }^{4}$ Individuals differ with respect to preferences and assignments. We thus exclude from our analysis further differences between individuals - i.e. household size, special needs, etc...-which would justify considering some individuals more deserving than others. The extension to such cases is left to future research.

${ }^{5}$ Here $x_{j}>x_{i}$ means that, in each dimension, $x_{j}$ is not larger than $x_{i}$ and that $x_{j} \neq x_{i}$.

${ }^{6}$ Individuals' preferences are assumed to be convex, ensuring that our transfer axiom does not conflict with efficiency.

${ }^{7}$ In a multicommodity setting, it seems natural for society to value progressive transfers between any two individuals, when one is assigned more of each commodity. When individuals have different preferences, however, this axiom clashes with the Pareto principle (see Fleurbaey and Trannoy (2003)). A transfer axiom similar to ours is discussed in Fleurbaey and Maniquet (2011). 
Three further requirements are standard in the literature. Weak Pareto requires social welfare to increase when all individuals are made better off. Continuity says that small changes of the allocation should not cause large jumps in the level of social welfare. Separability requires the ranking of two allocations to be independent of the assignment of an individual who is assigned the same bundle in both allocations.

The above six axioms uniquely characterize the class of what we call "opportunityequivalent utilitarian" welfare criteria. Each member of this class is uniquely identified by two objects.

The first object is a family of nested opportunity sets, where an opportunity set is a set of consumption bundles. The family can be parametrized by a function satisfying certain properties. The larger the value of the parameter, the larger the opportunity set to which it is associated. This value is then interpreted as the well-being reached by an individual who is assigned her most preferred bundle - or a bundle she finds equally desirable - in the opportunity set associated with this value. ${ }^{8}$ Whenever two individuals are assigned their preferred bundles from the same opportunity set, they are regarded as achieving the same well-being level. If one is assigned her preferred bundle from a larger opportunity set, she is regarded as achieving a higher well-being. These well-beings function are then subjected to a common "cardinalizing function," endogenously singled out by the axioms. ${ }^{9}$ It is the least concave function which ensures concavity of each individual's numerical representation of preferences. Then, the resulting well-being indices: (i) represent individuals' preferences, (ii) are interpersonally comparable; and (iii) are cardinally measurable.

The second object is an increasing and concave function that is applied to the profile of well-beings. This function reflects inequality aversion of the society and is standard for "generalized" versions of the utilitarian criterion (see Fleming (1952)).

The family of opportunity-equivalent utilitarian welfare criteria accommodates a large variety of views about justice. First, the characterization imposes only few restrictions on the family of nested opportunity sets: by changing the shape of opportunity sets, society can freely set the ethical importance and substitutability of commodities for each level of well-being. Among the special cases: when each opportunity set consists of the bundles that can be purchased (at fixed prices) at

\footnotetext{
${ }^{8} \mathrm{~A}$ similar broad concept of opportunity sets is analyzed by Thomson (1994): he suggests an allocation to be called equal-opportunity equivalent if each individual is indifferent between her assignment and her preferred bundle in the same opportunity set. In the present framework, such allocation is welfare maximizing if the society is infinitely inequality averse.

${ }^{9}$ Note that cardinal measurability - i.e. uniqueness up to an increasing affine transformation - is a necessary property of numerical representation of preferences for their sum to represent a well-defined social ranking of allocations.
} 
some wealth level, the money-metric representation of preferences emerges (McKenzie (1957) and Samuelson and Swamy (1974)); when each opportunity set consists of the bundles that are smaller than a fraction/multiple of a reference bundle, the "ray" representation of preferemces emerges (Pazner (1979)).

Next, commodities might represent different objects: functioning and capabilities (as suggested by Sen (1992)), resources (Dworkin (1981)), or a combination of primary goods and resources (Rawls (1971)). In each case, the shape of opportunity sets will generally vary depending on the commodities considered.

Finally, different degrees of inequality aversion can be introduced: the welfare criterion ranges from the simple sum of individuals' well-being indices - in Bentham's tradition - to attributing full priority to the worst-off individual in society - as suggested by Rawls (1971).

Beyond providing a fairness justification of utilitarianism, our results also shed new light on the dispute on ordinalist welfare analysis. Bergson (1938), and later Samuelson (1947), suggested that welfare criteria be based only on ordinal and noncomparable information about preferences. ${ }^{10}$ In the late 70 's, the hope to construct a social welfare function relying only on such information succumbed to the robustness of Arrow (1963)'s theorem (Kemp and Ng (1976) and Parks (1976)). The economic community considered settled the controversy and concluded that utility information is necessary to pass from individual tastes to social preferences, if social preferences are to reflect individual preferences without being dictatorial.

Few economists rejected this conclusion. Samuelson (1977) criticized the ethical appeal of the set of axioms imposed by Kemp and Ng (1976). He considered the social choice problem of distributing a stock of 100 chocolates between two individuals; for this example, he proposed a welfare criterion that is intuitive and only based on ordinal and non-comparable information about preferences, thus reaffirming the possibility of ordinalist welfare analysis. Mayston $(1974,1982)$ presented similar critiques, which led him to weaken Arrow's set of axioms. Based on these weaker axioms, he proposed a set of rules to generate ordinalist welfare criteria. Unfortunately, the extreme richness of these families of criteria provides little help to welfare analysis and, arguably, impeded his contributions to attract the attention of the general audience. In contrast, our family of criteria is more concrete, due to above-mentioned fairness axioms. Pazner (1979), and in particular Fleurbaey and Maniquet (2011), provided much sharper welfare criteria. Within specific eco-

\footnotetext{
${ }^{10}$ As Arrow (1963, p.109) writes, the ordinal informational basis is a grounding feature and key contribution of Bergson's approach: "It is the great merit of Bergson's 1938 paper to have carried the same [ordinalist] principle into the analysis of social welfare. The social welfare function [or welfare criterion] was to depend only on indifference maps..."
} 
nomic domains, they showed how to construct interpersonally comparable indices of well-being from fairness principles and each individual's indifference curve through the assigned bundles. However, by disregarding information about the remaining indifference curves, inequality aversion leads to a "Rawlsian" type of welfare criteria: no sacrifice for the worst-off is acceptable, independently of how large the benefits for all other individuals in society is. ${ }^{11}$ This information is not disregarded here. We show the role of indifference maps for identifying indices of well-being that are cardinally measurable and, thus, can avoid society to place no weight on individuals who are not the worst-off.

More recently, other authors have proposed ordinalist welfare criteria that are not of the Rawlsian type. Fleurbaey and Maniquet (2014) suggest a two-stage procedure: they first address the question of how to choose an interpersonally comparable numerical representation of individual preferences; they then invoke cardinal measurability of such representations and derive welfare criteria based on them. A first difference is that here cardinal measurability is endogenous. A further difference pertains to the domain of preference profiles over which social preferences are defined: we identify a family of welfare criteria that depend only on the preferences of the individuals in society; the criteria proposed by Fleurbaey and Maniquet, instead, are "universal," in that they depend on the preferences of individuals in all possible societies and are thus identical across societies. The same difference arises with Fleurbaey and Tadenuma (2014), who explicitly aim at comparing societies that may consist of different populations of individuals. ${ }^{12}$

The remainder of the paper is organized as follows. The next section illustrates the family of opportunity-equivalent utilitarian criteria. Section 3 presents the model and the axioms. Section 4 contains the main results. Section 5 concludes. Longer proofs are gathered in the appendix.

\footnotetext{
${ }^{11}$ Formally, they imposed the axiom of "independence of non-indifferent alternatives:" it requires the ranking of two alternatives to be unaffected by all changes of preferences, provided the indifference curves through the assigned bundles remain unchanged. Importantly, this axiom is weaker than Arrow's "independence of irrelevant alternatives:" which requires the ranking of two alternatives to be unaffected by all changes of preferences, provided the ranking of the assigned bundles remains unchanged. Interestingly, the intuition that information about preferences might be ethically relevant was already stated by Arrow (1967, p.19): "The potential usefulness of irrelevant alternatives is that they may permit empirically meaningful interpersonal comparisons," also clarifying that his earlier classification of such alternatives as "irrelevant" was misleading.

${ }^{12}$ Instead of weakening the information requirement, they avoid the Rawlsian type of criteria by further weakening the equity principle. The multicommodity progressive transfer is imposed only among individuals with the same and homothetic preferences. As a result, society might prefer to redistribute commodities from a worse-off individual to a better-off individual, when these have the same, but non-homothetic, preferences.
} 


\section{Utilitarianism in practice: a pedagogical example}

An economist analyses an economic model with two (equally-sized types of) individuals, $A$ and $B$. Individuals have preferences over consumption $c$ and leisure $(1-l)$, where $l$ denotes working time as a fraction of available time. Individuals' utilities are:

$$
u_{A} \equiv\left(c_{A}\right)^{\alpha}\left(1-l_{A}\right)^{1-\alpha} ; \quad u_{B} \equiv\left(c_{B}\right)^{\beta}\left(1-l_{B}\right)^{1-\beta} ;
$$

with $\alpha, \beta \in(0,1)$. The standard utilitarian criterion is $W \equiv u_{A}+u_{B}$.

The economist asks two data analysts, Dana and Terrence, to (i) estimate the parameters $\alpha$ and $\beta$ from individual data and (ii) apply the criterion $W$ to compute some optimal policy (which we leave unspecified). ${ }^{13}$ Dana and Terrence agree on their estimated utility parameters: $\hat{\alpha}=\frac{2}{3}$ and $\hat{\beta}=\frac{1}{3}$. Surprisingly, however, the optimal policies significantly differ.

The reason is soon discovered. Dana used consumption data in dollars, say $c^{D}$; while Terrence used consumption data in thousands of dollars, say $c^{T}$. It follows that Dana measures $A$ 's utility by $u_{A}^{D}=\left(c_{A}^{D}\right)^{\frac{2}{3}}\left(1-l_{A}\right)^{\frac{1}{3}}$, while Terrence by $u_{A}^{T}=$ $\left(c_{A}^{T}\right)^{\frac{2}{3}}\left(1-l_{A}\right)^{\frac{1}{3}}$. Since $u_{A}^{D}$ is an order preserving transformation of $u_{A}^{T}$, i.e. $u_{A}^{D}=$ $(1000)^{\frac{2}{3}} u_{A}^{T}$, both functions represent the same preferences. This explains the same estimates of $\alpha$ and, by a similar argument, of $\beta$.

The welfare criterion applied by Dana is then $W^{D} \equiv u_{A}^{D}+u_{B}^{D}$; the one applied by Terrence is $W^{T} \equiv u_{A}^{T}+u_{B}^{T}$. Substituting Terrence's utilities $u_{A}^{T}$ and $u_{B}^{T}$ in Dana's welfare criterion gives $W^{D}=(1000)^{\frac{2}{3}} u_{A}^{T}+(1000)^{\frac{1}{3}} u_{B}^{T}$. As the "welfare weights" $(1000)^{\frac{2}{3}}$ and $(1000)^{\frac{1}{3}}$ are different, the criterion $W^{D}$ represents different social preferences than $W^{T}$. Taking the ratio of these welfare weights, one discovers that $W^{D}$ attaches 10 times more importance to the well-being of individual $A$ than $W^{T}$. No wonder that the optimal policies differ!

This puzzle highlights a first difficulty with utilitarian practice. Utilities $u_{A}$ and $u_{B}$ are not accurately defined: the unit of measurement of commodities is left unspecified. This information is necessary for interpersonal comparability of well-beings and is crucial for a meaningful use of $W$. Moreover, as interpersonal comparability is an ethical choice, it should not be left to the data analysts.

By highlighting this problem, however, we only scratched the surface of a deeper

\footnotetext{
${ }^{13} \mathrm{How}$ preferences are estimated (or calibrated) is irrelevant for the discussion. For example, Aaberge and Colombino (2013) estimates individuals' numerical representation of preferences from individual budget sets and observed choices to address the design of optimal redistribution systems.
} 
issue: the implementation of a "utility-based" approach to utilitarianism. When formalizing the criterion $W$, the economist defends a specific way to (i) cardinally measure and (ii) interpersonally compare individual well-beings. In the following, we first criticize the economist's criterion $W$ (keeping aside the mentioned issue with the unit of measurement). We then illustrate how our approach endogenously derives cardinally measurable and interpersonally comparable representations of preferences.

To compare the allocations first-ranked by the different criteria, we close our model of the economy with the following technology. Output $y$ cannot exceed a linear function of total labor time: $y \leq 27\left(l_{A}+l_{B}\right) .{ }^{14}$ Individuals have no initial consumption and have available 1 unit of time; let $\alpha=\frac{2}{3}$ and $\beta=\frac{1}{3}$. At the Walrasian equilibrium, $A$ is assigned the bundle $\left(18, \frac{1}{3}\right)$, while $B$ is assigned $\left(9, \frac{2}{3}\right)$. Individuals' preferences are reflected in the equilibrium assignment: $A$ consumes more of the output, but enjoys less leisure; $B$ consumes less of the output, but enjoys more leisure. This assignment is particularly "egalitarian" as each individual is assigned her most preferred bundle from the same budget set. In particular, this allocation satisfies: Pareto optimality, i.e. it is not possible to make someone better off without making someone else worse off; and envy freeness, i.e. each individual is at least as well off at her assignment as at the assignment of anyone else.

For this economy, the criterion $W$ would assign consumption and leisure differently. It would optimally assign the bundle $\left(36, \frac{2}{3}\right)$ to individual $A$ and the bundle $(0,0)$ to individual $B$. This means that $B$ should work full-time for the benefit of $A$, who can enjoy all output. This recommendation is difficult to accept. However, utilitarianism is known to be insensitive to the distribution of well-beings across individuals. In fact, introducing enough inequality aversion avoids this recommendation. Let $f$ be a real-valued, increasing, and concave function; then, the "generalized utilitarian" criterion is $W^{f} \equiv f \circ u_{A}+f \circ u_{B}$, where $\circ$ denotes a composition of functions. As $f$ becomes more concave, a higher priority is assigned to the individuals with lower utilities. In the example, the more concave $f$ is, the higher the well-being of $B$. We argue next that, independently of the choice of $f, W^{f}$ is not a compelling welfare criterion.

To illustrate, compare the bundles that give to each individual the same utility

\footnotetext{
${ }^{14}$ The argument does not rely on the technology being linear. The equality of $A$ and $B$ 's marginal productivities of labor is instead important: the only differences between individuals are their preferences and the bundles they are assigned. When instead productivities differ, additional ethical issues arise: to which extent do individuals deserve these different productivities (i.e. due to education effort, luck, family background, etc...)? how does this affect the quantity of consumption and leisure each individual should be assigned? These questions are left to future research.
} 


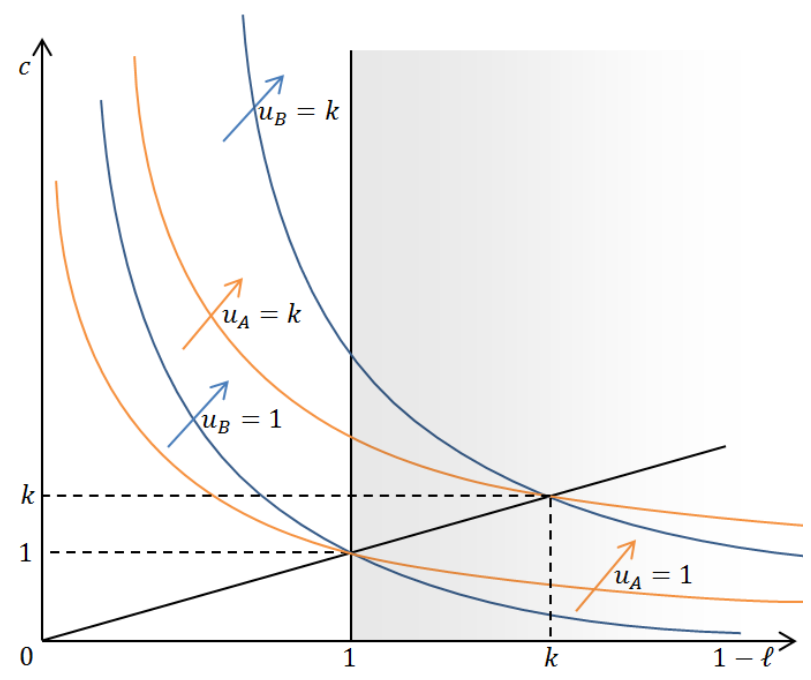

Figure 2.1: Individual $A$ is "cheaper to satisfy" than individual $B$.

level $u_{A}=u_{B}=k$. The indifference curves cross when $\left(c_{A}, 1-l_{A}\right)=\left(c_{B}, 1-l_{B}\right)=$ $(k, k)$. When $\left(1-\ell_{A}\right)=\left(1-\ell_{B}\right)>k$, individual $A$ requires a larger consumption than $B$ to reach utility level $k$. When $\left(1-\ell_{A}\right)=\left(1-\ell_{B}\right)<k$, individual $A$ requires a smaller consumption than $B$ to reach utility level $k$. In the first case, $B$ is "cheaper to satisfy" than $A$ : thus, in economies in which leisure is relatively abundant, $B$ is more effective in transforming commodities into utilities. In the second case, $A$ is "cheaper to satisfy" than $B$ : in economies in which leisure is relatively scarce, $A$ is more effective in transforming commodities into utilities.

This symmetry might suggest that $W$ is morally defensible: in a different economy in which output were relatively scarce, $W$ would optimally assign a larger bundle to $B$ than to $A$. Unfortunately, this is not the case here as the quantity of leisure enjoyable by each individual is bounded by 1 . Thus, whenever it is feasible for $B$ to achieve a utility $u_{B}=k>1$, leisure is necessarily smaller than $k$. Consequently, $A$ would be "cheaper to satisfy" than $B$ and $W$ discriminates against individual $B$. This is illustrated in Fig. 2.1. Since leisure is bounded by 1, for each feasible bundle at which individual $B$ achieves the utility level $u_{B}=k$, there exists a smaller bundle at which individual $A$ achieves the same utility level $u_{A}=k$. Thus, society achieves a higher social welfare $W$ by redistributing resources from individual $B$ to individual $A$. Our fairness axioms exclude this type of social preferences.

How about $W^{f}$ ? Inequality aversion introduces an opposite effect: when individuals are assigned the same bundle and $B$ achieves a lower utility, society gives $B$ a higher priority in the assignment of resources. While inequality aversion can counterbalance the effect of the comparability choice on the first-ranked allocation, the two distortions are different and do not cancel out. In fact, at the limiting case 
for the concavity of $f$ going to infinity, $W^{f}$ corresponds to the Rawlsian criterion $\min \left\{u_{A}, u_{B}\right\}$. This ranking is generally interpreted as the ranking of a perfectly egalitarian society. Nevertheless, it would here optimally assign the bundle $\left(9, \frac{1}{6}\right)$ to individual $A$ and the bundle $\left(\frac{27}{2}, 1\right)$ to individual $B$ : $A$ is the only one to work and, yet, $B$ should consume more of the output.

Our results suggest a fairness justification for the interpersonal comparability and cardinal measurability of the preference representations. As mentioned in the introduction, many normative viewpoints can be introduced by choosing: (i) an opportunity mapping and (ii) social inequality aversion. In the following, we provide an example based on money-metric representations of preferences. ${ }^{15}$

Each opportunity set consists of the bundles that can be purchased with wealth $w \geq 0$ at prices $p \in \mathbb{R}_{++}^{2}$. The opportunity mapping emerges by varying $w$. Let $p=(1,27)$ be the market prices arising at the Walrasian equilibrium above. Then for each $w \in \mathbb{R}_{+}$, the opportunity set $B_{w}$ is the set of bundles $(c, 1-l)$ such that $c+27(1-l) \leq w$. At any assignment $(c, 1-l)$, the well-being of an individual is $w$ if she is indifferent between $(c, 1-l)$ and her preferred bundle in $B_{w}$. Let such money-metric representation of preferences be $u_{A}^{m}$ and, similarly, $u_{B}^{m}$. These are easily computed and can be expressed in terms of $u_{A}$ and $u_{B}$ as follows:

$$
u_{A}^{m}=\left\{\begin{array}{ll}
2^{-\frac{2}{3}} 9 u_{A} & \text { if } u_{A} \leq 2^{\frac{2}{3}} 9 \\
u_{A}^{\frac{3}{2}}+27 & \text { otherwise }
\end{array} ; \quad u_{B}^{m}= \begin{cases}2^{-\frac{2}{3}} 27 u_{B} & \text { if } u_{B} \leq 2^{-\frac{1}{3}} 3 \\
u_{B}^{3}+27 & \text { otherwise }\end{cases}\right.
$$

This example is illustrated in Fig. 2.2. The opportunity set $B_{27}$ is the set of bundles available to individuals $A$ and $B$ at the Walrasian equilibrium. The Walrasian allocation gives $x_{A} \equiv\left(18, \frac{1}{3}\right)$ to individual $A$ and $x_{B} \equiv\left(9, \frac{2}{3}\right)$ to individual $B$. As these are their preferred bundles from the same set $B_{27}$, their index of well-being is $u_{A}^{m}=u_{B}^{m}=27$. When individual $A$ is assigned the bundle $\bar{x}_{A}$, her index of wellbeing is $u_{A}^{m}=27 / 2$ : she is indifferent between $\bar{x}_{A}$ and her preferred bundle from the opportunity set $B_{27 / 2}$. When individual $B$ is assigned the bundle $\bar{x}_{B}$, her index of well-being is $u_{B}^{m}=81 / 2$ : she is indifferent between $\bar{x}_{A}$ and her preferred bundle from the opportunity set $B_{81 / 2}$. Interestingly, $B$ 's preferred bundle in any larger opportunity set is a corner solution with $\left(1-\ell_{B}\right)=1$. This explains the non-linear relationship between $u_{B}^{m}$ and $u_{B}$ defined above.

The money-metric representations $u_{A}^{m}$ and $u_{B}^{m}$ are not considered cardinally mea-

\footnotetext{
${ }^{15}$ It is out of the scope of this paper to defend a specific ethical choice for the allocation of consumption and leisure across individuals. Related as well as alternative suggestions are advanced by Fleurbaey and Maniquet $(2006,2011)$ and empirically applied in Bargain et al. (2013).
} 


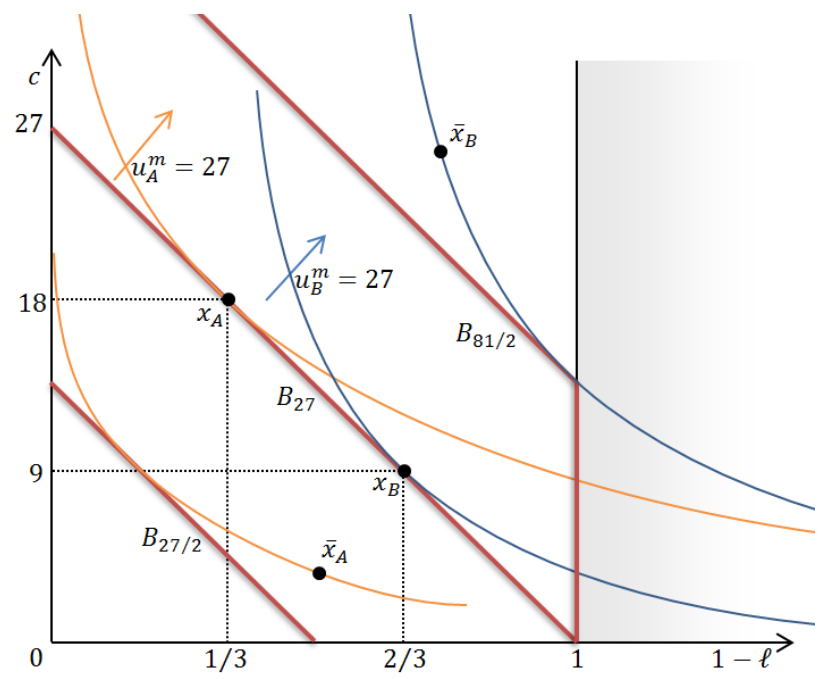

Figure 2.2: The construction of money-metric representations of preferences.

surable by our criteria. The reason is that these representations are not concave. Thus, a social ranking defined by their sum would recommend regressive redistributions of resources, i.e. from a worse-off to a better-off individual. ${ }^{16}$ Inequality-prone social preferences are excluded when a sufficiently concave transformation of $u_{A}^{m}$ and $u_{B}^{m}$ is introduced, as required by our fairness axioms.

The minimal transformation necessary to ensure concavity of the representations is a computational exercise. This function, denoted by $\phi$, ensures that $\phi \circ u_{A}^{m}$ and $\phi \circ u_{B}^{m}$ are concave and it is the minimally concave function that does so (see Debreu (1976)). ${ }^{17}$ Then, the cardinally measurable and interpersonally comparable representations of preferences are:

$$
\phi \circ u_{A}^{m}=\left\{\begin{array}{ll}
\frac{1}{3} u_{A} & \text { if } u_{A} \leq 2^{-\frac{1}{3}} 9 \\
\left(2^{-\frac{2}{3}} 9 u_{A}-27\right)^{\frac{1}{3}} & \text { if } 2^{-\frac{1}{3}} 9<u_{A} \leq 2^{\frac{2}{3}} 9 ; \\
\left(u_{A}\right)^{\frac{1}{2}} & \text { otherwise }
\end{array} \quad \phi \circ u_{B}^{m}=u_{B} .\right.
$$

Compared to $W$, the numerical representation of preferences is unchanged for $B$, while it has less weight and is more concave for $A$. The different weight follows

\footnotetext{
${ }^{16}$ Blackorby and Donaldson (1988) show that money-metrics are concave representations of homothetic preferences on $\mathbb{R}_{+}^{2}$. While here preferences are homothetic, leisure is constrained by 1 ; thus, their result does not hold. Since the Cobb-Douglas representation of homothetic preferences is a least concave representation, $u_{A}^{m}$ is not concave when $u_{A}>2^{\frac{2}{3}} 9$ and $u_{B}^{m}$ is not concave when $u_{B}>2^{-\frac{1}{3}} 3$.
}

${ }^{17}$ For the above example, $\phi$ is given by: $\phi(z)= \begin{cases}2^{\frac{2}{3}} 3^{-3} z & \text { if } z \leq \frac{81}{2} \\ (z-27)^{\frac{1}{3}} & \text { otherwise. }\end{cases}$ 
from the specific opportunities used to establish interpersonal comparability. The intuition for the concavity of $\phi \circ u_{A}^{m}$ goes as follows. Since preferences are homothetic, $u_{A}$ and $u_{B}$ are least concave representations of their preferences. The money-metric representations $u_{A}^{m}$ and $u_{B}^{m}$ are, instead, convex transformations of $u_{A}$ and $u_{B}$ and, thus, non-concave representations of preferences. The degree of convexity, however, differs among them: $u_{A}^{m}$ is less convex than $u_{B}^{m}$. Thus, when $\phi$ offsets the larger convexity of $u_{B}^{m}$, it inevitably leads to a representation of $A$ 's preferences that is more concave than $u_{A}$.

Finally, as in generalized versions of utilitarianism, further concavity can be introduced through function $f$. Then, the family of opportunity-equivalent utilitarian criteria characterized in this paper-for the above choice of opportunity sets - is:

$$
\bar{W}^{f} \equiv f \circ \phi \circ u_{A}^{m}+f \circ \phi \circ u_{B}^{m}
$$

When $f$ is linear, society is indifferent between all the efficient and interior distributions of resources. The choice of opportunities establishes comparability in a way that makes individuals equally effective in transforming commodities into well-being. Thus, society is indifferent between these assignments, suggesting that no individual is discriminated against. At the limit when $f$ is infinitely concave instead, society first-ranks the assignment corresponding to the Walrasian equilibrium above. By definition, $f$ reflects the social concern for inequalities in individuals' money-metrics. Thus, when money-metrics are equalized and maximized, each individual is assigned her preferred alternative from the same opportunity set.

In synthesis, our approach: (i) establishes interpersonal comparability and avoids discrimination across individuals through the choice of opportunity sets; (ii) endogenously singles out the minimal concavity needed for cardinal measurability (thorugh the function $\phi$ ); and (iii) allows society to set the preferred degree of inequality aversion $f$.

\section{The framework and the axioms}

\subsection{The framework}

A society consists of a non-empty and finite set of individuals $N=\{1, \ldots, n\}$ with $n \geq 3$. Each individual $i \in N$ is characterized by a preference relation $R_{i} ; R_{i}$ is a weak order on the $\ell$-dimensional Euclidean commodity space $X=\mathbb{R}_{+}^{\ell}$, with $\ell$ finite. ${ }^{18}$ The strict preference and indifference relations induced by $R_{i}$ are denoted

\footnotetext{
$\overline{18 \mathbb{R}_{+}^{\ell} \text { denotes the non-negative orthant }}$ of $\mathbb{R}^{\ell}$. Vector inequalities are denoted $>, \geq$, and $\gg$.
} 
by $P_{i}$ and $I_{i}$. Each preference relation $R_{i}$ can be represented by a strictly increasing and concave numerical function. Each type of preferences is shared by at least two individuals, that is, for each $i \in N$, there exists $j \in N \backslash\{i\}$ such that $R_{i}=R_{j}$. We discuss these assumptions below.

An allocation $x \equiv\left(x_{1}, \ldots, x_{n}\right) \in X^{n}$ assigns a bundle $x_{i}$ to each individual $i \in N$. For each $i \in I$ and each $x_{i} \in X$, let $e^{i}\left(x_{i}\right) \in X^{n}$ be the allocation that assigns $x_{i}$ to individual $i$ and the zero bundle to all other individuals. Then, each allocation $x \equiv\left(x_{1}, \ldots, x_{n}\right) \in X^{n}$ can be written as $x=\sum_{i \in N} e^{i}\left(x_{i}\right)$. A social ranking, denoted $\succsim$, is a weak order of allocations. For each pair of allocations $x, x^{\prime} \in X^{n}$, $x \succsim x^{\prime}$ means that $x$ is at least as socially desirable as $x^{\prime}$. The strict social preference and indifference relations induced by $\succsim$ are denoted by $\succ$ and $\sim$.

Remark 1 . The commodity space $X$ can be generalized to any convex subset of $\mathbb{R}_{+}^{\ell}$ such that there exists a bundle that is considered worst by all individuals. In $\mathbb{R}_{+}^{\ell}$, this worst bundle is the zero vector.

Remark 2. Preference relations that admit a concave numerical representation are convex, but not all convex preference relations admit a concave numerical representation. As we show in Subsection 4.5, our axioms are mutually compatible only if preference relations are representable by concave functions. Nevertheless, this is not a very restrictive assumption as every convex preference relations can be approximated by a sequence of preference relations that admit concave numerical representations (Kannai (2004)). ${ }^{19}$

Remark 3. We require each preference relation to be shared by at least two individuals. This richness assumption ensures that a later-introduced fairness axiominvolving exclusively individuals with the same preference relation - is non-vacuous. While simplifying the exposition, the same conclusions can be derived without this asssumption. In a multi-profile framework, it can be replaced by a replication invariance axiom. ${ }^{20}$ The social ranking should be unchanged when comparing two allocations for a specific society and when comparing the replicas of these allocations for the replicas of these societies.

\footnotetext{
${ }^{19}$ Moreover, smooth and strictly convex preference relations defined on compact sets-a framework to which our results extend - always admit concave numerical representations (Mas-Colell (1985)).

${ }^{20}$ In a multi-profile framework, the social ranking needs to be defined for each admissible society (defined by the preference profile of individuals). The replication invariance axiom is an interprofile condition which relates the social ranking for different societies. See d'Aspremont and Gevers (2002).
} 


\subsection{Basic axioms}

The next three axioms are standard in the literature. First, if each individual prefers her assignment at $x \in X^{n}$ to her assignment at $x^{\prime} \in X^{n}$, then $x$ is socially preferred to $x^{\prime}$.

Weak Pareto: For each pair $x, x^{\prime} \in X^{n}, x_{i} P_{i} x_{i}^{\prime}$ for each $i \in N$ implies $x \succ x^{\prime}$.

Next, small changes in allocations do not cause large jumps in social welfare.

Continuity: For each $x \in X^{n}$, the sets $\left\{x^{\prime} \in X^{n} \mid x^{\prime} \succsim x\right\}$ and $\left\{x^{\prime} \in X^{n} \mid x \succsim x^{\prime}\right\}$ are closed.

Finally, the ranking of two alternatives is independent of the bundle assigned to an individual who is unconcerned by the choice. ${ }^{21}$

Separability: For each pair $x, x^{\prime} \in X^{n}$, if there is $i \in N$ such that $x_{i}=x_{i}^{\prime} \equiv a_{i}$, then for each $b_{i} \in X$,

$$
\left(a_{i}, x_{-i}\right) \succsim\left(a_{i}, x_{-i}^{\prime}\right) \Longleftrightarrow\left(b_{i}, x_{-i}\right) \succsim\left(b_{i}, x_{-i}^{\prime}\right)
$$

\subsection{Fairness axioms}

The social concern for fairness is embodied in three very intuitive axioms.

The first axiom limits the influence that any individual can exert on the social ranking. Let $i \in N$ and $x_{i} \in X$. We require that, for each $j \in N$, there exists a bundle $x_{j} \in X$ such that $e^{j}\left(x_{j}\right) \in X^{n}$ is socially indifferent to $e^{i}\left(x_{i}\right)$. Society can then trade-off the well-being of any two individuals, ruling out dictatorial welfare criteria. $^{22}$

Possibility of trade-offs: For each pair $i, j \in N$ and each $x_{i} \in X$, there exists

$$
x_{j} \in X \text { such that } e^{j}\left(x_{j}\right) \sim e^{i}\left(x_{i}\right) .
$$

The next axiom prevents a certain form of discrimination among individuals. Let $i \in N$ and $x_{i}^{*} \in X$. Then, $i$ is discriminated against if, for each $x_{i} \in X$ such that $x_{i} I_{i} x_{i}^{*}$, society is worse off at allocation $e^{i}\left(x_{i}\right)$ than at any allocation $e^{j}\left(x_{j}\right) \in X^{n}$ which assigns a smaller bundle $x_{j}<x_{i}$ to some individual $j \in N \backslash\{i\}$. In other

\footnotetext{
${ }^{21}$ The following notation is standard: for each $x \in X^{n}$, each $i \in N$, and each $a_{i} \in X$, let $\left(a_{i}, x_{-i}\right) \in X^{n}$ be the allocation that assigns $a_{i}$ to $i$ and $x_{j}$ to each $j \in N \backslash\{i\}$.

${ }^{22}$ This axiom is inspired by "weak anonymity" introduced in d'Aspremont (1985) (see also d'Aspremont and Gevers (2002)). Our axiom is stronger. Weak anonymity requires that for each pair of individuals $i, j \in N$ there exist two allocations $x^{i}, x^{j} \in X^{n}$ such that: $x_{i}^{i} P_{i} x_{i}^{j}$; $x_{j}^{j} P_{j} x_{j}^{i}$; and $x_{k}^{i} I_{k} x_{k}^{j}$ for each $k \neq i, j$.
} 
words, individual $i$ is discriminated against if there is no bundle on her indifference curve through $x_{i}^{*}$ which society would prefer $i$ to consume instead of giving less to some other individual. Such discrimination is prevented here for each $i \in I$ and each $x_{i}^{*} \in X .^{23}$

Non Discrimination: For each $i \in N$ and each $x_{i}^{*} \in X$, there exists $x_{i} \in X$ with $x_{i} I_{i} x_{i}^{*}$ such that for each $j \in N \backslash\{i\}$ and each $x_{j} \in X, e^{j}\left(x_{j}\right) \succ e^{i}\left(x_{i}\right)$ implies $x_{j} \nless x_{i}$.

With one commodity only, say income, non discrimination is weaker than anonymity. Society is indifferent to income permutations on the subset of distributions which assign a positive income to at most one individual. More generally, non discrimination avoids society to consider some individual more deserving than the others. ${ }^{24}$

The last axiom is related to the Pigou-Dalton transfer principle. Interpreting a justice concern expressed by Pigou (1912), Dalton (1920) suggests that a progressive transfer from a richer to a poorer individual (provided the richer/poorer relation is not reversed) leads to a more desirable distribution of income. As in Fleurbaey and Maniquet (2011), we extend the transfer to multiple commodities and restrict it to individuals with the same preferences.

Equal-Preference Transfer: For each pair $j, k \in N$ such that $R_{j}=R_{k} \equiv R_{0}$, if there exist a pair $x, x^{\prime} \in X^{n}$ and $\alpha \geq 0$ such that: $x_{j}-\alpha\left(x_{j}-x_{k}\right)=x_{j}^{\prime} R_{0} x_{k}^{\prime}=x_{k}+\alpha\left(x_{j}-x_{k}\right) ;$

then for each $i \in N \backslash\{j, k\}, x_{i}=x_{i}^{\prime}$; $x^{\prime} \succsim x$.

${ }^{23}$ This axiom is related to "convex hull inclusion," introduced by Fleurbaey and Maniquet (2014) to address the measurement of individual well-beings. Let an individual's upper-contour at her assignment be the set of bundles she considers at least as desirable as her assignment. According to their axiom, individual $i$ achieves a higher well-being than individuals $j$ and $k$ if her upper-contour set at her assignment is contained in the interior of the convex hull of the upper-contour sets of these individuals at their assignments. Combined with weak Pareto, continuity and separability, our axiom remains weaker: it requires individual $i$ to achieve a higher well-being than individuals $j$ and $k$ when $i$ 's upper-contour set at her assignment is in the interior of the union - a subset of the convex hull — of the upper-contours of these individuals at their assignments.

${ }^{24}$ This is related to the problem of expensive tastes, summarized by Sen (1979, p.203) as follows: "if person $\mathrm{A}$ as a cripple gets half the utility that the pleasure-wizard person $\mathrm{B}$ does from any given level of income, then in the pure distribution problem between A and B the utilitarian would end up giving the pleasure-wizard B more income than the cripple A." In our approach, information is restricted to individuals' ordinal preferences: no information is available about the capacity of individuals to transform resources into utilities. Thus, non discrimination impedes society to arbitrarily consider some individuals "cripples" and others "pleasure-wizards." 
The axiom reads as follows. Individuals $j, k \in N$ have the same preferences $R_{j}=$ $R_{k} \equiv R_{0}$. At allocation $x \in X^{n}$, individual $j$ is assigned a more desirable bundle than individual $k$ according to $R_{0}$. At $x^{\prime} \in X^{n}$, the difference between assignments is reduced by a transfer that is proportional to $x_{j}-x_{k}$; importantly, $j$ remains at least as well off as $k$ after the transfer, i.e. $x_{j}^{\prime} R_{0} x_{k}^{\prime}$. Since all other individuals are unaffected, the after-transfer allocation has less inequality and is considered at least as socially desirable as the initial one.

\section{The main result}

In this section, we define our welfare criteria and present our characterization.

\subsection{The opportunity-equivalent utilitarian criterion}

We construct the interpersonally comparable and cardinally measurable numerical representation of each individual's preferences in two steps. The first step identifies ordinal level comparability: society considers individuals equally well-off when they are assigned "equivalent opportunities." The second step establishes cardinal measurability and builds on the idea of a least-concave representation of preferences, introduced by Debreu (1976).

An opportunity mapping $C$ identifies a family of nested consumption sets (Thomson (1994)). For each $\lambda \in \mathbb{R}_{+}, C(\lambda) \subseteq X$ is the opportunity set of "size" $\lambda$ : it is non-empty, closed, and satisfies free disposal (for each pair $c, c^{\prime} \in X$ with $c \geq c^{\prime}$, $\left.c \in C(\lambda) \Rightarrow c^{\prime} \in C(\lambda)\right)$. Also, $C(0)=\{0\}$ and $\lim _{\lambda \rightarrow \infty} C(\lambda)=X$. Moreover, $C$ is continuous and "strongly monotonic" (for each pair $\lambda, \lambda^{\prime} \in \mathbb{R}_{+}$with $\lambda^{\prime}>\lambda$ and each $x \in C(\lambda)$ there exists $x^{\prime} \gg x$ such that $x^{\prime} \in C\left(\lambda^{\prime}\right)$ ). Let $\mathcal{C}$ be the set of opportunity mappings. For each $i \in N$ and each $\bar{X} \subseteq X$, let $m_{i}(\bar{X})$ denote the set of bundles that maximize preferences $R_{i}$ in $\bar{X}$.

Consider an opportunity mapping $C \in \mathcal{C}$. Individual $i$ is assigned a bundle $x_{i} \in X$. Select $\lambda_{i} \in \mathbb{R}_{+}$such that individual $i$ is indifferent between her preferred alternative in the set $C\left(\lambda_{i}\right)$ and $x_{i}$ or, more formally, satisfies $x_{i} I_{i} z_{i}$ with $z_{i} \in m_{i}\left(C\left(\lambda_{i}\right)\right)$. Then, $\lambda_{i}$ can be interpreted as a measure of the well-being of individual $i$ at $x_{i}$.

The role of the opportunity mapping $C$ for interpersonal comparisons of well-being is illustrated in Fig. 4.1 for individuals $i$ and $j$. Individual $i$ is assigned the bundle $x_{i}$, which is for her indifferent to her preferred alternative in $C\left(\lambda_{i}\right)$. Individual $j$ is assigned the bundle $x_{j}$, which is for her indifferent to her preferred alternative in $C\left(\lambda_{j}\right)$. When comparing the assignments $x_{i}$ and $x_{j}$, what matters is the size of these opportunity sets $C\left(\lambda_{i}\right)$ and $C\left(\lambda_{j}\right)$. As $\lambda_{i}>\lambda_{j}$, individual $i$ is considered 


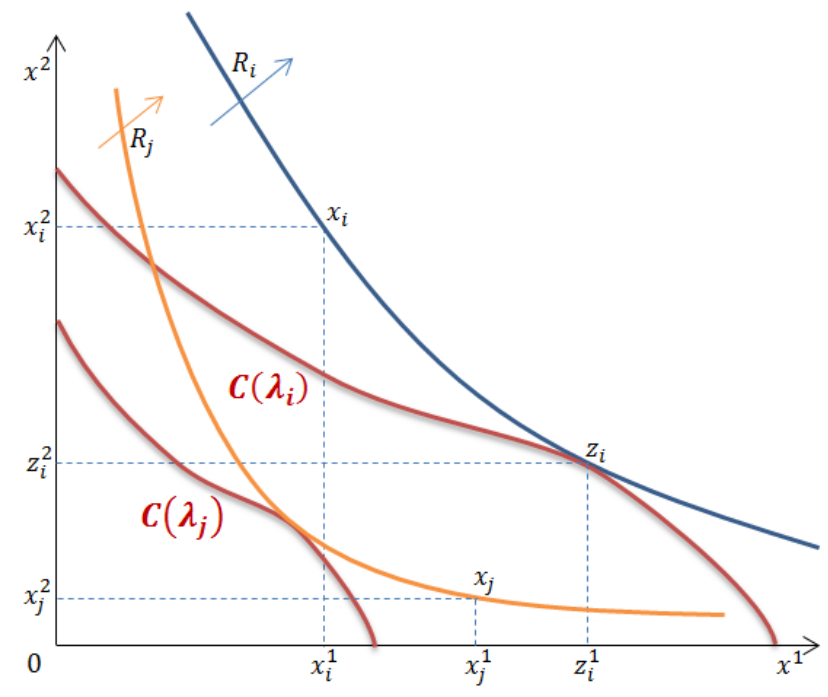

Figure 4.1: The opportunity-equivalent well-being function.

better off than individual $j$. Importantly, such comparisons are not affected by order preserving transformations of the well-being measure. ${ }^{25}$

Formally, for each $i \in N$, the opportunity-equivalent well-being function relative to $C \in \mathcal{C}$ is the function $U_{i}^{C}: X \rightarrow \mathbb{R}$ defined by setting for each $x_{i} \in X$ :

$$
U_{i}^{C}\left(x_{i}\right) \equiv \lambda_{i} \Longleftrightarrow x_{i} I_{i} z_{i} \text { with } z_{i} \in m_{i}\left(C\left(\lambda_{i}\right)\right)
$$

As we shall see, society makes interpersonal comparisons of well-being through the functions $\left(U_{i}^{C}\right)_{i \in N}$ for some choice of opportunity mapping $C \in C$. For each $i \in N, U_{i}^{C}$ is a continuous numerical representation of preferences $R_{i}$. Given an opportunity mapping $C \in \mathcal{C}$ and the corresponding opportunity-equivalent wellbeing functions $\left(U_{i}^{C}\right)_{i \in N}$, we can now construct the indices of well-being that society considers cardinally measurable. Define $\Psi^{C}$ as the set of all real-valued functions $\psi: \mathbb{R} \rightarrow \mathbb{R}$ such that, for each $i \in N, \psi \circ U_{i}^{C}$ is a concave representation of preferences $R_{i}$. Let $\Phi^{C} \subset \Psi^{C}$ be the set of least joint concave transformations: $\phi \in \Phi^{C}$ implies that for each $\psi \in \Psi^{C}, \psi$ is more concave than $\phi .{ }^{26}$ Then, society considers

${ }^{25}$ Conversely, the choice of $C \in \mathcal{C}$ matters for the identification of the worse-off individual. Nevertheless, it is not identified by the characterization result and is a free ethical choice. This is unsurprising: the shape of opportunity sets defines the ethical importance and substitutability of commodities for each level of well-being. Even restricting to opportunities for welfare delimited by a linear function (that is budget sets), this would require specifying the relative prices of commodities: this cannot be chosen in an abstract setting, where each commodity could equally represent a standard consumption good, a basic right, or a capability.

${ }^{26} \mathrm{~A}$ function $s$ is more concave than a function $s^{\prime}$ if there exist a real-valued and concave transformation $g$ such that $s=g \circ s^{\prime}$. Least concave numerical representations of preferences are studied in Debreu (1976) and Kannai (1977). These are later applied to measure risk (Kihlstrom and Mirman (1981)), inequality (Atkinson and Bourguignon (1982) and Bosmans et al. (2015)), 
interpersonally comparable and cardinally measurable the transformed opportunityequivalent well-being functions $\left(\phi \circ U_{i}^{C}\right)_{i \in N}$ for some $\phi \in \Phi^{C}$. We next show that such transformations exist. The proof is provided in the appendix.

Lemma 1. For each opportunity mapping $C \in \mathcal{C}$, the set $\Phi^{C}$ is nonempty.

The social welfare criterion can now be defined. A social ranking $\succsim$ is opportunityequivalent utilitarian if there exists $C \in \mathcal{C}, \phi \in \Phi^{C}$, and an increasing and concave function $f$, such that $\succsim$ is represented by the function $W: X^{n} \rightarrow \mathbb{R}$, defined by setting, for each $x \in X^{n}$,

$$
W(x)=\sum_{i \in N} f \circ \phi \circ U_{i}^{C}\left(x_{i}\right)
$$

Each addend requires the specification of three objects:

(i) $U_{i}^{C}$ is the representation of preferences that society assigns to individual $i$ to make interpersonal comparisons of well-being. These functions are uniquely determined by the opportunity mapping $C \in \mathcal{C}$. At bundle $x_{i}$, the level of well-being achieved by $i$ is the size $\lambda_{i}$ of the opportunity set $C\left(\lambda_{i}\right)$ that makes her indifferent between her assignment $x_{i}$ and her preferred bundle in $C\left(\lambda_{i}\right)$.

(ii) $\phi \in \Phi^{C}$ adds cardinal measurability to the representation of preferences. As $\Phi^{C}$ consists of all increasing affine transformations of $\phi$, the choice of $\phi \in \Phi^{C}$ is irrelevant for the social ranking. The set $\Phi^{C}$ is uniquely determined by the opportunity mapping $C \in \mathcal{C}$. The function $\phi$ guarantees that for each individual $i \in N, \phi \circ U_{i}^{C}$ is concave. Furthermore, it is among the least concave transformations that do so.

(iii) $f$ is a real-valued and concave function. The concavity of $f$ reflects inequality aversion. The more concave $f$ is, the more society wants to redistribute from individuals who are considered better off to individuals who are considered worse off. At the limit, as concavity tends to infinity, society assigns absolute priority to the individual with the lowest well-being.

In the one-commodity case - say income - the opportunity-equivalent well-being functions are identical across individuals. The common opportunity-equivalent wellbeing $U^{C}$ can be any increasing function of income. Since in the one-commodity

and poverty (Atkinson (2003)). To preserve interpersonal comparability of the opportunity equivalent well-beings, we derive the least joint concave representations of preferences: the transformation ensuring concavity of the representations is jointly imposed on all individuals. A related idea is discussed in Mandler (2006): to compromise between ordinality and cardinality of utility information, he suggests taking as primitives all the increasing and concave transformations of ordinally comparable utilities and develops a partial order based on joint concavity. 
case, the least concave representation of a strictly monotone preference relation is linear, $\phi$ is the inverse of $U^{C}$. The family of criteria we characterize is then the sum of any concave transformation - identified by function $f$ - of each individual's income level. This solution corresponds to the "non-utility-based" justification of utilitarianism, discussed in the introduction.

\subsection{The characterization result}

Our main result is that a social ranking satisfies the axioms introduced above if and only if it is opportunity-equivalent utilitarian. The proof is discussed in the next subsections.

Theorem 1. A social ranking satisfies weak Pareto, continuity, separability, possibility of trade-offs, non discrimination, and equal-preference transfer if and only if it is opportunity-equivalent utilitarian.

This result shows that the "non-utility-based" justification of the welfare criterion in eq. (1) has a counterpart in multicommodity settings. Income is replaced by a measure of access to opportunity sets. Concavity of the numerical representation of preferences is ensured by the least joint concave transformation of the opportunityequivalent well-being functions. Social inequality aversion may be added. More importantly, Theorem 1 shows that interpersonal comparability and cardinal measurability of individuals' preferences need not be exogenously assumed.

Before illustrating the proof, it is worthwhile presenting the formulation of the criteria that emerges when preferences and opportunity sets have a particularly simple form. A preference relation $R_{i}$ is homothetic if for each $\alpha>0$ and each pair $x, x^{\prime} \in X, x I x^{\prime}$ implies $\alpha x I \alpha x^{\prime}$. Let $\mathcal{V}_{i}$ be the set of numerical representations of preferences $R_{i}$ that are homogeneous of degree 1 . The proof of the existence of such representations is immediate and omitted. An opportunity mapping $C \in \mathcal{C}$ is homothetic if for each pair $\lambda, \lambda^{\prime}>0$, there exists $\alpha>0$ such that $x \in C(\lambda)$ if and only if $\alpha x \in C\left(\lambda^{\prime}\right)$.

Theorem 2. Let preferences be homothetic. Assume there exists a homothetic opportunity mapping $C \in \mathcal{C}, \phi \in \Phi^{C}$, and an increasing and concave function $f$ such that the social ranking $\succsim$ can be represented by the function $W: X^{n} \rightarrow \mathbb{R}$, defined by setting, for each $x \in X^{n}$,

$$
W(x)=\sum_{i \in N} f \circ \phi \circ U_{i}^{C}\left(x_{i}\right)
$$


For each $i \in N$ and some $\lambda^{*}>0$, let $z_{i} \in m_{i}\left(C\left(\lambda^{*}\right)\right)$ and $V_{i} \in \mathcal{V}_{i}$. Then, $\succsim$ can be represented by the function $\bar{W}: X^{n} \rightarrow \mathbb{R}$, defined by setting, for each $x \in X$ :

$$
\bar{W}(x)=\sum_{i \in N} f \circ \frac{V_{i}\left(x_{i}\right)}{V_{i}\left(z_{i}\right)} .
$$

The proof is in the appendix. Intuitively, when preferences are homothetic, any least concave representation is homogeneous of degree 1 . Moreover, by homotheticity of the opportunity mapping, an individual's preferred bundle from one opportunity set is proportional to (one of) her preferred bundle from any other opportunity set. As a consequence, interpersonal comparability and cardinal measurability can be simply recovered by normalizing each individual $i$ 's function $V_{i}$ with respect to the well-being achieved at a reference opportunity set, here $C\left(\lambda^{*}\right)$. Thus, for each individual $i \in N$, this normalized well-being $\frac{V_{i}\left(x_{i}\right)}{V_{i}\left(z_{i}\right)}$ substitutes $\phi \circ U_{i}^{C}\left(x_{i}\right)$ in the representation of the opportunity-equivalent utilitarian social ranking.

\subsection{Properties of the opportunity-equivalent utilitarian social ranking}

Here, we show that the opportunity-equivalent utilitarian social ranking satisfies the axioms we introduced, thus proving the "if" part of Theorem 1.

Lemma 2. If a social ranking is opportunity-equivalent utilitarian, then it satisfies weak Pareto, continuity, separability, possibility of trade-offs, non discrimination, and equal-preference transfer.

Proof. Let $\succsim$ be an opportunity-equivalent utilitarian social ranking. Let $C \in \mathcal{C}$ and let $W$ represent $\succsim$. Verifying the first three axioms is immediate. By construction, $W$ is increasing with respect to some numerical representation of each individual's preferences. Thus, it satisfies weak Pareto. Continuity of the functions $f, \phi$, and $\left(U_{i}^{C}\right)_{i \in N}$ ensures continuity. Furthermore, $W$ is additive with respect to some numerical representation of each individual's preferences. Thus, separability holds.

We next show that possibility of trade-offs is satisfied. Let $i \in N$ and $x_{i} \in$ $X$. By construction of $U_{i}^{C}$, individual $i$ is indifferent between $x_{i}$ and her preferred bundle in $C\left(U_{i}^{C}\left(x_{i}\right)\right)$, i.e. $x_{i} I_{i} m_{i}\left(C\left(U_{i}^{C}\left(x_{i}\right)\right)\right)$. Let $j \in N \backslash\{i\}$ and $x_{j} \in m_{j}\left(C\left(U_{i}^{C}\left(x_{i}\right)\right)\right)$. Then, $U_{j}^{C}\left(x_{j}\right)=U_{i}^{C}\left(x_{i}\right)$. Moreover, for each $k \in N$, $U_{k}^{C}(0)=0$. Thus, $W\left(e^{i}\left(x_{i}\right)\right)=f \circ \phi \circ U_{i}^{C}\left(x_{i}\right)=f \circ \phi \circ U_{j}^{C}\left(x_{j}\right)=W\left(e^{j}\left(x_{j}\right)\right)$, equivalently, $e^{j}\left(x_{j}\right) \sim e^{i}\left(x_{i}\right)$. 
To show that $\succsim$ satisfies non discrimination, let $i \in N$ and $x_{i}^{*} \in X$. By construction, $U_{i}^{C}\left(x_{i}^{*}\right)$ is such that $z_{i} \in m_{i}\left(C\left(U_{i}^{C}\left(x_{i}^{*}\right)\right)\right)$ satisfies $z_{i} I_{i} x_{i}^{*}$. Let $X_{i} \subset X$ be the set of bundles $x_{i} \in X$ such that $x_{i} I_{i} x_{i}^{*}$. Non discrimination is violated if for each $x_{i} \in X_{i}$, there exist $j \neq i$ and $x_{j}<x_{i}$ such that $e^{j}\left(x_{j}\right) \succsim e^{i}\left(x_{i}\right)$. This is impossible as $e^{j}\left(x_{j}\right) \succsim e^{i}\left(z_{i}\right)$ with $x_{j}<z_{i}$ requires $x_{j}$ to be $j$ 's preferred alternative in an opportunity set that contains $z_{i}$, contradicting monotonicity of preferences.

Finally, the concavity of $f \circ \phi \circ U_{i}^{C}$ for each $i \in N$ implies that $\succsim$ satisfies equal preference transfer. Formally, consider a progressive transfer between individuals $j$ and $k$ such that $R_{j}=R_{k} \equiv R_{0}$. Before the transfer, $j$ and $k$ are assigned $x_{j} \in X$ and $x_{k} \in X$ with $x_{j} P_{0} x_{k}$. Let $\alpha \geq 0$ be such that, after the transfer, $j$ and $k$ are assigned $x_{j}^{\prime}=x_{j}-\alpha\left(x_{j}-x_{k}\right)$ and $x_{k}^{\prime}=x_{k}+\alpha\left(x_{j}-x_{k}\right)$ with $x_{j}^{\prime} R_{0} x_{k}^{\prime}$. Let the remaining individuals be assigned the same bundles at $x$ and $x^{\prime}$; by additivity of $W$, their assignments are irrelevant for the ranking of $x$ and $x^{\prime}$. By definition of concavity,

$$
\begin{aligned}
& \phi \circ U_{0}^{C}\left(x_{j}^{\prime}\right) \geq(1-\alpha) \phi \circ U_{0}^{C}\left(x_{j}\right)+c \quad \alpha \phi \circ U_{0}^{C}\left(x_{k}\right), \\
& \phi \circ U_{0}^{C}\left(x_{k}^{\prime}\right) \geq \alpha \phi \circ U_{0}^{C}\left(x_{j}\right)+(1-\alpha) \phi \circ U_{0}^{C}\left(x_{k}\right) .
\end{aligned}
$$

Taking the sum, it follows that $W\left(x^{\prime}\right) \geq W(x)$, equivalently, $x^{\prime} \succsim x$.

\subsection{From the axioms to the opportunity-equivalent utilitarian social ranking}

In this subsection, we illustrate the welfare implications of the axioms. As an intermediate step, we introduce an anonymity requirement with respect to opportunity sets and show its relationship with the axioms introduced above. We start with two definitions.

A permutation is a bijection $\pi$ defined on the set of individuals. Denote by $\Pi$ the set of all permutations.

For each $C \in \mathcal{C}$, each $i \in N$ and each $\lambda \in \mathbb{R}_{+}$, individual $i$ is said to attain the opportunity set $\boldsymbol{C}(\boldsymbol{\lambda})$ if she is assigned a bundle $x_{i} \in X$ such that $x_{i} \in m_{i}(C(\lambda))$.

The next axiom reinterprets the "Suppes indifference" axiom on social rankings in our multicommodity setting. ${ }^{27}$ It requires society to be indifferent between permuting attained opportunities across individuals. We illustrate the idea in Fig. 4.2. Consider an opportunity mapping $C \in \mathcal{C}$. At allocation $x \in X^{n}$, individual $i \in N$ attains the opportunity set $C\left(\lambda_{i}\right)$, while individual $j$ attains the opportunity set

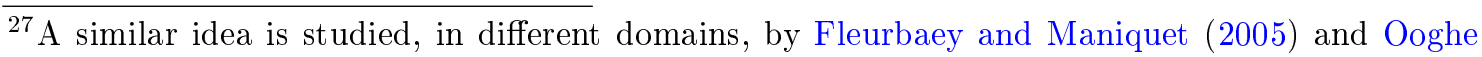
et al. (2007) and is inspired by Suppes (1966).
} 


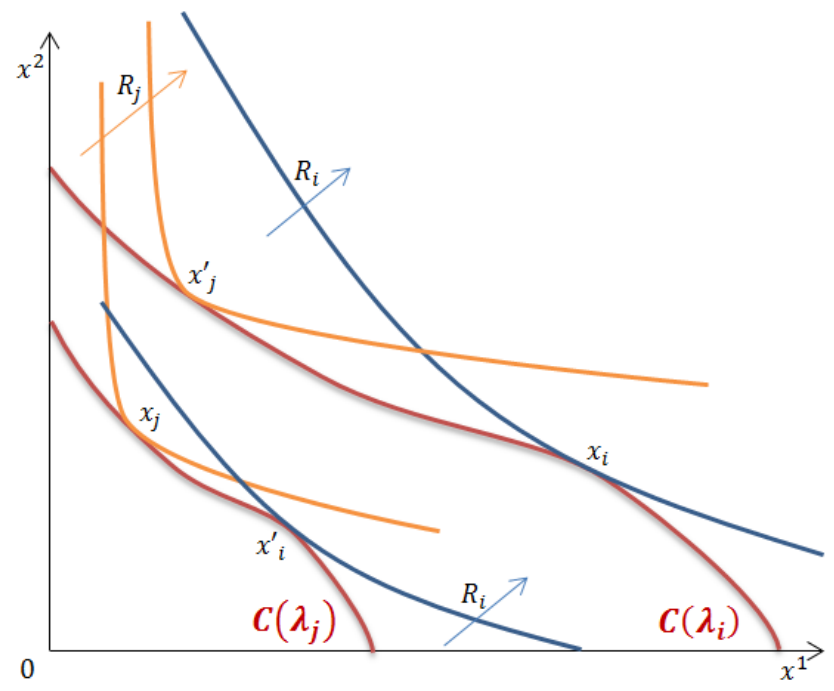

Figure 4.2: Representation of $C$-anonymous opportunities.

$C\left(\lambda_{j}\right)$. At allocation $x^{\prime}$, the opportunity sets that individuals $i$ and $j$ attain are permuted. Individual $i$ is now assigned the bundle $x_{i}^{\prime}$, which is her preferred bundle in $C\left(\lambda_{j}\right)$. Individual $j$ is instead assigned the bundle $x_{j}^{\prime}$, which is her preferred bundle in $C\left(\lambda_{i}\right)$. Assume each other individual $k \in N \backslash\{i, j\}$ attains an unchanged opportunity set $C\left(\lambda_{k}\right)$ at both allocations. Then, society should be indifferent between $x$ and $x^{\prime}$. Let $C \in \mathcal{C}$.

$C$-Anonymous Opportunities: For each pair $x, x^{\prime} \in X^{n}$ and each $\pi \in \Pi$, if for each $i \in N, x_{i} \in m_{i}\left(C\left(\lambda_{i}\right)\right)$ with $\lambda_{i} \in \mathbb{R}_{+}$and $x_{i}^{\prime} \in m_{i}\left(C\left(\lambda_{\pi(i)}\right)\right)$, then $x^{\prime} \sim x$.

In one-commodity economic environments, say income, $C$-anonymous opportunities simplifies to the classical version of anonymity and the function $C \in \mathcal{C}$ is immaterial. Each opportunity set is a compact interval $[0, \bar{c}]$ with $\bar{c} \in \mathbb{R}_{+}$. The opportunity mapping is obtained by varying $\bar{c}$. By monotonicity of preferences, each individual $i$ 's preferred income is the largest in the opportunity set, that is $\bar{c}=m_{i}([0, \bar{c}])$. Thus, permuting the opportunity sets individuals attain is equivalent to permuting their assigned incomes.

The next lemma shows the relationship between $C$-anonymous opportunities and the previous axioms.

Lemma 3. Let a social ranking $\succsim$ satisfy weak Pareto, continuity, separability, and possibility of trade-offs. Then, $\succsim$ satisfies non discrimination if and only if there exists $C \in \mathcal{C}$ such that $\succsim$ satisfies $C$-anonymous opportunities. 
The proof is in the appendix. ${ }^{28}$ That $C$-anonymous opportunities leads to non discrimination is rather unsurprising. We discuss here the reverse implication, needed to prove Theorem 1. Intuitively, by possibility of trade-offs, there exist $n$ socially indifferent allocations, each assigning a non-zero bundle to only one individual, this individual differing from allocation to allocation. For each individual, consider her lower contour set at her assigned bundle. The intersection across individuals of these lower contour sets is the set of bundles that all individuals consider at most as desirable as their assignment. This set is an opportunity set $C(\lambda)$ for some opportunity mapping $C \in \mathcal{C}$ and $\lambda \in \mathbb{R}_{+}$. By non discrimination, each individual is indifferent between her assignment and her preferred bundle in $C(\lambda)$. By weak Pareto and continuity, whenever all individuals are indifferent between the bundles they are assigned at two allocations, so is society. By transitivity, society is indifferent between all allocations at which one individual attains $C(\lambda)$ and all others are assigned the zero bundle. Applying the same reasoning to all sets of $n$ socially indifferent allocations allows us to construct the entire opportunity mapping $C \in \mathcal{C}$. By possibility of trade-offs and non discrimination, society is indifferent between allocations at which same-preference individuals attain permuted opportunity sets. By separability, this indifference extends to permutations across individuals with different preferences.

The axiom of $C$-anonymous opportunities plays a central role in establishing comparability across individuals. Weak Pareto, continuity, separability, and possibility of trade-offs force social preferences to be represented by a sum of numerical representations of individual preferences. Within this additive class, $C$-anonymous opportunities requires individuals' assignments to be compared in terms of the opportunity-equivalent well-being functions. Cardinal measurability and, thus, the opportunity-equivalent utilitarian criteria follow from equal-preference transfer. This is the content of the next lemma. The proof is in the appendix.

Lemma 4. Let $C \in \mathcal{C}$. If a social ranking $\succsim$ satisfies weak Pareto, continuity, separability, equal preference transfer, and $C$-anonymous opportunities, then there exist $\phi \in \Phi^{C}$ and an increasing and concave function $f$ such that $\succsim$ is represented by the function $W: X^{n} \rightarrow \mathbb{R}$, defined by setting, for each $x \in X^{n}$,

$$
W(x)=\sum_{i \in N} f \circ \phi \circ U_{i}^{C}\left(x_{i}\right)
$$

Lemmas 3 and 4 together imply the following result and complete the proof of

${ }^{28}$ The result in the appendix is slightly stronger: weak Pareto, continuity, separability, and C-anonymous opportunities imply minimal individual symmetry and non discrimination. 
Theorem 1.

Lemma 5. If a social ranking satisfies weak Pareto, continuity, separability, possibility of trade-offs, non discrimination, and equal-preference transfer, then it is opportunity-equivalent utilitarian.

\subsection{Domain maximality}

Following the approach of Barbera et al. (1991) and Ching and Serizawa (1998), we show that we cannot extend our preference domain and relax our assumption that preferences admit a concave representation.

Let $R \equiv\left(R_{i}\right)_{i \in N}$ be a preference profile. Let $\mathcal{R}$ be the domain of preference profiles adopted. For memory, each preference profile $R \in \mathcal{R}$ satisfies: (a) for each $i \in N, R_{i}$ can be represented by strictly increasing and concave numerical function; (b) for each $i \in N$, there exists $j \in N \backslash\{i\}$ such that $R_{i}=R_{j}$. A different domain of preferences $\overline{\mathcal{R}}$ can be defined. Each preference profile $R \in \overline{\mathcal{R}}$ satisfies: $(\bar{a})$ for each $i \in N, R_{i}$ is a weak order on $X$ that is continuous, strictly monotonic, and convex;

(b) for each $i \in N$, there exists $j \in N \backslash\{i\}$ such that $R_{i}=R_{j}$.

Domain maximality: A domain $\mathcal{R}^{*}$ is a maximal domain for a set of axioms if:

(i)

(ii)

(iii)

$$
\mathcal{R}^{*} \subseteq \overline{\mathcal{R}}
$$

there exists a social ranking satisfying the axioms on $\mathcal{R}^{*}$; there exists no domain $\mathcal{R}^{+}$such that $\mathcal{R}^{*} \subset \mathcal{R}^{+} \subseteq \overline{\mathcal{R}}$ and such that there exists a social ranking satisfying the axioms on $\mathcal{R}^{+}$.

The following result shows that our domain of preferences is a maximal domain for the opportunity-equivalent utilitarian criterion. The results holds even without imposing non discrimination. The proof is in the appendix.

Theorem 3. The domain $\mathcal{R}^{n}$ is a maximal domain for weak Pareto, continuity, separability, possibility of trade-offs, and equal-preference transfer.

\section{Concluding remarks}

It is a widespread opinion in the economics profession that no policy-relevant and ethically appealing welfare analysis can be performed without interpersonal comparability and cardinal measurability of utilities. Consequently, these assumptions are widely accepted, regardless of their weak empirical basis. 
This paper provides a new normative justification of utilitarianism, which only requires ordinal and non comparable information about preferences. According to the family of opportunity-equivalent utilitarian criteria that we obtain, individuals' well-beings are measured by endogenously selected "utility functions" that: (i) numerically represent individuals' preferences; (ii) are interpersonally comparable; (iii) are cardinally measurable; and (iv) are concave in the assignment. Social welfare is measured by the sum of a concave transformation of such well-beings.

The standard axioms of weak Pareto, continuity, and separability force social welfare to be additive with respect to some (weighted) numerical representation of each individual's preferences.

Interpersonal comparability builds on two intuitive fairness principles: possibility of trade-offs limits the influence of each individual in pairwise comparisons; and non discrimination says that no individual is considered to be more deserving than any other. These principles require to choose a family of nested opportunity sets, that is, an opportunity mapping. This choice establishes interpersonal comparability: when individuals are assigned their most preferred bundles from the same opportunity set, they can be understood as achieving the same well-being level.

Cardinal measurability is endogenously established by introducing inequality aversion among individuals with the same preferences. The fairness requirement of equal-preference transfer is a generalization of Dalton's transfer principle to multicommodity spaces. A crucial role is played by each individual's indifference map: the cardinal representation relates distances between indifference curves to differences in well-being levels and ensures concavity of each individual's measure of well-being.

Interpersonal comparisons are known to be unavoidable to judge conflicting alternatives. Yet, the economist need not assume comparability exogenously. Importantly, the mechanical exercise of maximizing the sum of some utility functions requires an evaluation of the underlying comparability and measurability assumptions. In models with different-preference individuals, it is not infrequent that the policy conclusion in favor of one group of individuals is (at least partially) explained by the favorable treatment of that group with respect to the definition of comparability. In fact, apparently innocuous assumptions on parametric forms of utilities might introduce discriminations among individuals and undermine the appeal of utilitarianism.

The results of this paper open many avenues for future research. First, theories of justice originally in stark contrast with utilitarianism (as those introduced by Rawls, Dworkin, or Sen) might instead be reconciled with it by an appropriate choice of commodities and opportunity mappings. Second, the implications of opportunity- 
equivalent utilitarianism for sensible economic domains need to be analyzed and can be empirically implemented; examples include public goods provision (related to Maniquet and Sprumont $(2004,2005)$ ) and income taxation (see Fleurbaey and Maniquet (2006) and Saez and Stantcheva (2015)), but also health provision, choices with risk, intergenerational investments and savings, etc... Third, more general and non-utilitarian classes of criteria for preference aggregation can be studied by relaxing the continuity and separability requirements. Fourth, multidimensional poverty and inequality measures can be derived from each such criterion and can be used to formulate policy recommendations.

Acknowledgments. The author thanks Rolf Aaberge, Geir Asheim, Claude d'Aspremont, Bård Harstad, Aanund Hylland, Edwin Leuven, Francois Maniquet, Frikk Nesje, and, particularly, William Thomson. This paper is part of the research activities of the Centre for the Study of Equality, Social Organization, and Performance (ESOP), supported by the Research Council of Norway, project number 179552. The research leading to these results has received funding from the ERC grant agreement n. 283236 (FP7/2007-2013).

\section{A. Proofs}

\section{Proof of Lemma 1}

Proof. Let $C \in \mathcal{C}$ and $i \in I$. By assumption, there exists a concave function $u_{i}: X \rightarrow \mathbb{R}$ that represents individual $i$ 's preferences $R_{i}$. As $U_{i}^{C}$ also represents preferences $R_{i}$, there exists a real-valued function $\psi$ such that $u_{i}=\psi \circ U_{i}^{C}$. Thus $\Psi^{C}$ is non empty. The non-emptiness of a set of least concave functions $\Phi^{C}$ follows directly from Debreu (1976).

\section{Proof of Theorem 2}

Proof. Let $i \in N, x_{i} \in X$, and $\alpha>0$. By definition of homogeneity of degree 1 , $V_{i}\left(\alpha x_{i}\right)=\alpha V_{i}\left(x_{i}\right)$. As $V_{i}\left(\alpha x_{i}\right)$ is linear with respect to $\alpha, V_{i}$ is a least concave representation of $R_{i}$. Let $\phi_{i}$ be a real-valued and strictly increasing function such that for each $x_{i} \in X, \phi_{i} \circ U_{i}^{C}\left(x_{i}\right)=\frac{V_{i}\left(x_{i}\right)}{V_{i}\left(z_{i}\right)}$. Since preferences and the opportunity mapping are homothetic, for each $\alpha \geq 0$ there exists $\lambda \geq 0$ such that $\alpha z_{i} \in m_{i}(C(\lambda))$. Thus, $\phi_{i} \circ U_{i}^{C}\left(\alpha z_{i}\right)=\frac{V\left(\alpha z_{i}\right)}{V\left(z_{i}\right)}$ implies that, for each $\alpha \geq 0, \phi_{i} \circ \lambda=\alpha$.

As this holds for each $i \in N, \phi_{i}$ is equal across individuals: let $\bar{\phi} \equiv \phi_{i}$. Since for each $i \in N, \bar{\phi} \circ U_{i}^{C}\left(x_{i}\right)=\frac{V_{i}\left(x_{i}\right)}{V_{i}\left(z_{i}\right)}$ and $\frac{V_{i}\left(x_{i}\right)}{V_{i}\left(z_{i}\right)}$ is a least concave representation of 
preferences, $\bar{\phi} \in \Phi^{C}$. As $\phi, \bar{\phi} \in \Phi^{C}, \bar{\phi}$ is an increasing affine transformation of $\phi$. Thus, $\bar{W}$ is ordinally equivalent to $W$ and represents social preferences $\succsim$.

\section{Proof of Lemma 3}

We first introduce some intermediate steps and then prove Lemma 3 in two parts.

The following axiom requires society to be indifferent between any two allocations whenever all individuals are.

Pareto indifference: For each pair $x, x^{\prime} \in X^{n}$, if $x_{i} I_{i} x_{i}^{\prime}$ for each $i \in N$, then

$$
x \sim x^{\prime} .
$$

Given the Euclidean structure of the commodity space and the completeness of the social ranking, weak Pareto and continuity imply Pareto indifference. This result is well-known and its proof is omitted.

We next show that weak Pareto, continuity, separability, and possibility of tradeoffs lead to a social ranking that can be represented by a function that is additive with respect to individual preferences. ${ }^{29}$

Lemma 6. If a social ranking $\succsim$ satisfies weak Pareto, continuity, separability, and possibility of trade-offs then for each $i \in N$ there exists a numerical representation of preferences $R_{i}$, say $v_{i}: X \rightarrow \mathbb{R}$, such that for each pair $x, x^{\prime} \in X^{n}$ :

$$
x \succsim x^{\prime} \Leftrightarrow \sum_{i \in N} v_{i}\left(x_{i}\right) \geq \sum_{i \in N} v_{i}\left(x_{i}^{\prime}\right)
$$

Proof. By assumption, $n \geq 3$. Furthermore, $\succsim$ is complete, transitive, and satisfies continuity and separability. By Gorman (1968)'s theorem on overlapping separable sets, there exist a continuous and strictly increasing function $h: \mathbb{R} \rightarrow \mathbb{R}$ and, for each $i \in N$, a continuous function $v_{i}: X \rightarrow \mathbb{R}$, such that for each pair $x, x^{\prime} \in X^{n}$ :

$$
x \succsim x^{\prime} \Leftrightarrow h\left[\sum_{i \in N} v_{i}\left(x_{i}\right)\right] \geq h\left[\sum_{i \in N} v_{i}\left(x_{i}^{\prime}\right)\right] .
$$

By weak Pareto and strict monotonicity of preferences, there exists $j \in N$ such that $\frac{\partial v_{j}}{\partial x_{j}} \neq 0$. By possibility of trade-offs and the additive representation of eq. (2), it follows that for each $i \in N, \frac{\partial v_{i}}{\partial x_{i}} \neq 0$. By weak Pareto, for each $i \in N, v_{i}$ represent preferences $R_{i}$. Since $h$ is ordinally irrelevant, the result follows.

\footnotetext{
${ }^{29}$ The same conclusion would hold when the social ranking satisfies continuity, separability, and the strong version of the Pareto principle. Strong Pareto cannot, however, substitute possibility of trade-offs for the conclusion of Theorem 1 and Lemma 3. Possibility of trade-offs further prevents that a well-being level is associated to a finite assignment for some individual, while the same well-being level requires an infinite assignment for some other individual.
} 
We finally show that, within the class of additive social rankings (given by Lemma 6), non discrimination implies anonymity among individuals with the same preferences.

Equal-Preference Anonymity: For each pair $j, k \in N$ such that $R_{j}=R_{k}$ and each pair $x, x^{\prime} \in X^{n}$, if $x_{k}=x_{j}^{\prime}, x_{j}=x_{k}^{\prime}$, and $x_{i}=x_{i}^{\prime}$ for each $i \neq j, k$, then $x \sim x^{\prime}$.

Lemma 7. If a social ranking $\succsim$ satisfies weak Pareto, continuity, separability, possibility of trade-offs, and non discrimination, then it also satisfies equal-preference anonymity.

Proof. Let $j, k \in N$ be such that $R_{j}=R_{k} \equiv R_{0}$. By Lemma 6, for each pair $x, x^{\prime} \in X^{n}, x \succsim x^{\prime}$ if and only if $\sum_{i \in N} v_{i}\left(x_{i}\right) \geq \sum_{i \in N} v_{i}\left(x_{i}^{\prime}\right)$, where, for each $i \in I$, $v_{i}$ is a numerical representation of preferences $R_{i}$. For each $i \in N$, let $\bar{v}_{i}: X \rightarrow \mathbb{R}$ be such that for each $x_{i} \in X, \bar{v}_{i}\left(x_{i}\right)=v_{i}\left(x_{i}\right)-v_{i}(0)$ : clearly $\bar{v}_{i}\left(x_{i}\right)$ represents preferences $R_{i}$ and $\sum_{i \in N} \bar{v}_{i}\left(x_{i}\right)$ represents the social ranking $\succsim$.

Let $x_{j}, x_{k} \in X$ be such that $x_{j} I_{0} x_{k}$. We first show that $e^{j}\left(x_{j}\right) \sim e^{k}\left(x_{k}\right)$. By contradiction, assume that $e^{j}\left(x_{j}\right) \succ e^{k}\left(x_{k}\right)$. Since for each $i \in I, \bar{v}_{i}(0)=0$, $\bar{v}_{j}\left(x_{j}\right)>\bar{v}_{k}\left(x_{k}\right)$. By continuity, for each $y_{k} \in X$ such that $y_{k} I_{0} x_{k}$ there exists $y_{j} \in X$ with $y_{j} \ll y_{k}$ such that $\bar{v}_{j}\left(x_{j}\right)>\bar{v}_{j}\left(y_{j}\right)>\bar{v}_{k}\left(y_{k}\right)=\bar{v}_{k}\left(x_{k}\right)$. Since $\bar{v}_{j}\left(y_{j}\right)>$ $\bar{v}_{k}\left(y_{k}\right)$ implies $e^{j}\left(y_{j}\right) \succ e^{k}\left(y_{k}\right)$, a contradiction with non discrimination follows. Thus, $\bar{v}_{j}\left(x_{j}\right)=\bar{v}_{k}\left(x_{k}\right)$ and, since this holds for each pair $x_{j}, x_{k} \in X$ such that $x_{j} I_{0} x_{k}$, also $\bar{v}_{j}=\bar{v}_{k}$.

Let $x, x^{\prime} \in X^{n}$ be such that $x_{k}=x_{j}^{\prime}, x_{j}=x_{k}^{\prime}$, and, for each $i \in N \backslash\{j, k\}$, $x_{i}=x_{i}^{\prime}$. Since $\bar{v}_{j}=\bar{v}_{k}, \sum_{i \in N} \bar{v}_{i}\left(x_{i}\right)=\sum_{i \in N} \bar{v}_{i}\left(x_{i}^{\prime}\right)$ and, equivalently, $x \sim x^{\prime}$. Thus, equal-preference anonymity is satisfied.

\section{Part 1}

We show that weak Pareto, continuity, separability, and $C$-anonymous opportunities imply possibility of trade-offs and non discrimination. For possibility of trade-offs, we present a stronger result, involving only Pareto indifference, instead of weak Pareto, continuity, and separability.

Lemma 8. Let $C \in \mathcal{C}$. If a social ranking $\succsim$ satisfies Pareto indifference and $C$-anonymous opportunities, then it satisfies possibility of trade-offs.

Proof. Let $R \in \mathcal{R}^{n}, i, j \in N$, and $x_{i} \in X$. Since $C$ is continuous, there exist $z_{i} \in X$ and $\lambda_{i} \geq 0$ such that $x_{i} I_{i} z_{i}$ and $z_{i} \in m_{i}\left(C\left(\lambda_{i}\right)\right)$. By Pareto indifference, $e^{i}\left(z_{i}\right) \sim e^{i}\left(x_{i}\right)$. Let $x_{j} \in m_{j}\left(C\left(\lambda_{i}\right)\right)$. Since, for each $k \in I, 0=m_{k}(C(0))$, $C$-anonymous opportunities implies $e^{i}\left(z_{i}\right) \sim e^{j}\left(x_{j}\right)$, where $i$ and $j$ have access to 
permuted opportunity sets. By transitivity, $e^{i}\left(x_{i}\right) \sim e^{j}\left(x_{j}\right)$. Since this holds for each $x_{i} \in X$ and each $j \neq i$, satisfies possibility of trade-offs.

Lemma 9. Let $C \in \mathcal{C}$. If a social ranking $\succsim$ satisfies weak Pareto, continuity, separability, and $C$-anonymous opportunities, then it also satisfies non discrimination.

Proof. The proof is by contraposition. Suppose that $\succsim$ violates non discrimination. Then, there exist $i \in N$ and $x_{i}^{*} \in X$ such that for each $x_{i} \in X$ with $x_{i} I_{i} x_{i}^{*}$, there are $j \neq i$ and $x_{j} \in X$ for which $e^{j}\left(x_{j}\right) \succ e^{i}\left(x_{i}\right)$ and $x_{j} \leq x_{i}$. Let $z_{i} \in X$ and $\lambda_{i} \geq 0$ be such that $z_{i} I_{i} x_{i}^{*}$ and $z_{i} \in m_{i}\left(C\left(\lambda_{i}\right)\right)$. Let $j \in N$ and $x_{j} \in X$ be such that $e^{j}\left(x_{j}\right) \succ$ $e^{i}\left(z_{i}\right)$ and $x_{j} \leq z_{i}$. Let $z_{j} \in m_{j}\left(C\left(\lambda_{i}\right)\right)$. Since $m_{i}(C(0))=0, C$-anonymous opportunities implies $e^{i}\left(z_{i}\right) \sim e^{j}\left(z_{j}\right)$. By transitivity, $e^{j}\left(x_{j}\right) \succ e^{j}\left(z_{j}\right)$. Let $\lambda_{j} \geq 0$ be such that $x_{j} \in m_{j}\left(C\left(\lambda_{j}\right)\right)$. Since $x_{j} \leq z_{i}$ and $z_{i} \in m_{i}\left(C\left(\lambda_{i}\right)\right)$, then $\lambda_{i} \geq \lambda_{j}$.

Case 1: $\lambda_{i}=\lambda_{j}$. Then, $z_{j} I_{j} x_{j}$. Thus, $e^{j}\left(x_{j}\right) \succ e^{j}\left(z_{j}\right)$, contradicting Pareto indifference (implied by weak Pareto and continuity).

Case 2: $\lambda_{i}>\lambda_{j}$. Then, $z_{j} P_{j} x_{j}$. By Lemma $6, e^{j}\left(x_{j}\right) \succ e^{j}\left(z_{j}\right)$ is equivalent to $v_{j}\left(x_{j}\right)>v_{j}\left(z_{j}\right)$, which violates monotonicity of $R_{j}$.

\section{Part 2}

We show that weak Pareto, continuity, separability, possibility of trade-offs, and non discrimination imply that there exists $C \in \mathcal{C}$ such that $C$-anonymous opportunities is satisfied.

Proof. Step 1. For each $i \in N$ and each $x_{i} \in X$, let the lower- and uppercontour-sets of $i$ at $x_{i}$ be defined as $L C S_{i}\left(x_{i}\right) \equiv\left\{x \in X \mid x_{i} R_{i} x\right\}$ and $U C S_{i}\left(x_{i}\right) \equiv$ $\left\{x \in X \mid x R_{i} x_{i}\right\}$.

Let $x_{i} \in X$. For each $\lambda \geq 0$, let $x_{i}(\lambda) \in X$ be such that $x_{i}(\lambda)=\lambda x_{i}$. By possibility of trade-offs, for each $j \in N \backslash\{i\}$ and each $\lambda \geq 0$, there exists $x_{j} \in X$ such that $e^{i}\left(x_{i}(\lambda)\right) \sim e^{j}\left(x_{j}\right)$. Let then

$$
C(\lambda) \equiv\left\{x \in X \mid x \in \bigcap_{j \in N} \operatorname{LCS}_{j}\left(x_{j}\right)\right\} .
$$

By monotonicity and continuity of preferences, $C(\lambda)$ is non-empty, closed, and satisfies free disposal. Moreover, $C(0)=\{0\}$ and $\lim _{\lambda \rightarrow \infty} C(\lambda)=X$. By continuity of preferences, $C$ is continuous with respect to $\lambda$. By monotonicty of preferences, $\lambda>\lambda^{\prime}$ implies that for each $x^{\prime} \in C\left(\lambda^{\prime}\right)$ there exists $x \in C(\lambda)$ with $x \gg x^{\prime}$. Thus, $C \in \mathcal{C}$. 
Step 2. We show that if for some $j \in N$ and some $\lambda \geq 0, U C S_{j}\left(x_{j}(\lambda)\right) \cap C(\lambda)=$ $\emptyset$, then non discrimination is violated.

If this is the case, for each $\bar{x}_{j} \in X$ such that $\bar{x}_{j} I_{j} x_{j}(\lambda)$, there exist $k \in N \backslash\{j\}$ and $\bar{x}_{k} \in X$ with $\bar{x}_{k}<\bar{x}_{j}$ and $\bar{x}_{k} I_{k} x_{k}(\lambda)$. By Pareto indifference (implied by weak Pareto and continuity), $e^{j}\left(\bar{x}_{j}\right) \sim e^{j}\left(x_{j}(\lambda)\right)$ and $e^{k}\left(\bar{x}_{k}\right) \sim e^{k}\left(x_{k}(\lambda)\right)$. By construction, $e^{j}\left(x_{j}(\lambda)\right) \sim e^{k}\left(x_{k}(\lambda)\right)$. By transitivity, $e^{j}\left(\bar{x}_{j}\right) \sim e^{k}\left(\bar{x}_{k}\right)$. This is a violation of non discrimination.

Thus, for each $j \in N$ and each $\lambda \geq 0, U C S_{j}\left(x_{j}(\lambda)\right) \bigcap C(\lambda) \neq \emptyset$. Furthermore, for each $j \in N$, each $\lambda \geq 0$, and each $\hat{x}_{j} \in m_{j}(C(\lambda))$, we have $x_{j}(\lambda) I_{j} \hat{x}_{j}$ and, by Pareto indifference, $e^{j}\left(x_{j}(\lambda)\right) \sim e^{j}\left(\hat{x}_{j}\right)$.

Step 3. Let $\underline{\lambda}<\bar{\lambda}, i, j \in N$, and $\underline{x}_{i}, \bar{x}_{j} \in X$ be such that $\underline{x}_{i} \in m_{i}(C(\underline{\lambda}))$ and $\bar{x}_{j} \in m_{j}(C(\bar{\lambda}))$. Let $\bar{x}_{i}, \underline{x}_{j} \in X$ be such that $\bar{x}_{i} \in m_{i}(C(\bar{\lambda}))$ and $\underline{x}_{j} \in m_{j}(C(\underline{\lambda}))$. We show that $e^{i}\left(\underline{x}_{i}\right)+e^{j}\left(\bar{x}_{j}\right) \sim e^{i}\left(\bar{x}_{i}\right)+e^{j}\left(\underline{x}_{j}\right)$. Two cases can occur.

Case 1: $R_{i}=R_{j}$. By equal-preference anonymity (see Lemma 7),

$$
e^{i}\left(\underline{x}_{i}\right)+e^{j}\left(\bar{x}_{j}\right) \sim e^{i}\left(\bar{x}_{j}\right)+e^{j}\left(\underline{x}_{i}\right) .
$$

By equal preferences, for each $\lambda \geq 0, m_{i}(C(\lambda))=m_{j}(C(\lambda))$ and, thus, $\bar{x}_{j} I_{i} \bar{x}_{i}$ and $\underline{x}_{i} I_{j} \underline{x}_{j}$. By Pareto indifference, $e^{i}\left(\underline{x}_{i}\right)+e^{j}\left(\bar{x}_{j}\right) \sim e^{i}\left(\bar{x}_{i}\right)+e^{j}\left(\underline{x}_{j}\right)$.

Case 2: $R_{i} \neq R_{j}$. By assumption, there exists $\ell \in N \backslash\{i, j\}$ such that $R_{\ell}=R_{i}$. By previous definitions:

$$
\begin{aligned}
e^{\ell}\left(x_{\ell}(\bar{\lambda})\right) & \sim e^{j}\left(x_{j}(\bar{\lambda})\right), \\
e^{\ell}\left(x_{\ell}(\underline{\lambda})\right) & \sim e^{j}\left(x_{j}(\underline{\lambda})\right) .
\end{aligned}
$$

Note that $\bar{x}_{j} I_{j} x_{j}(\bar{\lambda})$ and $\bar{x}_{i} I_{\ell} x_{\ell}(\bar{\lambda}) \in m_{\ell}(C(\bar{\lambda}))$; correspondingly, $\underline{x}_{j} I_{j} x_{j}(\underline{\lambda})$ and $\underline{x}_{i} I_{\ell} x_{\ell}(\bar{\lambda}) \in m_{\ell}(C(\underline{\lambda}))$. Thus, by Pareto indifference:

$$
\begin{aligned}
e^{\ell}\left(\bar{x}_{i}\right) & \sim e^{j}\left(\bar{x}_{j}\right), \\
e^{\ell}\left(\underline{x}_{i}\right) & \sim e^{j}\left(\underline{x}_{j}\right) .
\end{aligned}
$$

By separability:

$$
\begin{aligned}
e^{i}\left(\underline{x}_{i}\right)+e^{\ell}\left(\bar{x}_{i}\right) & \sim e^{i}\left(\underline{x}_{i}\right)+e^{j}\left(\bar{x}_{j}\right), \\
e^{i}\left(\bar{x}_{i}\right)+e^{\ell}\left(\underline{x}_{i}\right) & \sim e^{i}\left(\bar{x}_{i}\right)+e^{j}\left(\underline{x}_{j}\right) .
\end{aligned}
$$

By equal-preference anonymity,

$$
e^{i}\left(\underline{x}_{i}\right)+e^{\ell}\left(\bar{x}_{i}\right) \sim e^{i}\left(\bar{x}_{i}\right)+e^{\ell}\left(\underline{x}_{i}\right) .
$$


By transitivity,

$$
e^{i}\left(\underline{x}_{i}\right)+e^{j}\left(\bar{x}_{j}\right) \sim e^{i}\left(\bar{x}_{i}\right)+e^{j}\left(\underline{x}_{j}\right) .
$$

Thus, society is indifferent between permuting access to opportunity sets among any two individuals. By separability and transitivity, this extends to permutations among any number of individuals. Thus, $C$-anonymous opportunities holds.

\section{Proof of Lemma 4}

The result is established in several lemmas.

Let a social ranking $\succsim$ be additively representable if for each $i \in N$ there exists a function $v_{i}$ representing preferences $R_{i}$, such that for each pair $x, \bar{x} \in X^{n}$, $x \succsim \bar{x} \Leftrightarrow \sum_{i \in N} v_{i}\left(x_{i}\right) \geq \sum_{i \in N} v_{i}\left(\bar{x}_{i}\right)$. Let $W(x) \equiv \sum_{i \in N} v_{i}\left(x_{i}\right)$. By Lemma 6 , weak Pareto, continuity, separability, and possibility of trade-offs require $\succsim$ to be additively representable.

Lemma 10. If a social ranking $\succsim$ is additively representable and satisfies equalpreference transfer, then for each pair $j, k \in N$ with $R_{j}=R_{k}, v_{j}$ and $v_{k}$ are (i) concave; and (ii) equal up to an additive constant, i.e. $v_{j}=v_{k}+c$ for some $c \in \mathbb{R}$.

Proof. Let $x, x^{\prime} \in X^{n}$ be such that for some $j, k \in N$ with $R_{j}=R_{k} \equiv R_{0}$ and some $\alpha \geq 0$ the following statements hold:

(i) $x_{j}^{\prime}=x_{j}-\alpha\left(x_{j}-x_{k}\right) R_{0} x_{k}+\alpha\left(x_{j}-x_{k}\right)=x_{k}^{\prime}$;

(ii) $x_{i}=x_{i}^{\prime}$ for each $i \in N \backslash\{j, k\}$.

By equal-preference transfer, $x^{\prime} \succsim x$ and, by additive representability:

$$
v_{j}\left(x_{j}^{\prime}\right)+v_{k}\left(x_{k}^{\prime}\right) \geq v_{j}\left(x_{j}\right)+v_{k}\left(x_{k}\right) .
$$

Rearrange, substitute $x_{j}^{\prime}$ and $x_{k}^{\prime}$, and divide by $\alpha$. Taking the limit for $\alpha \rightarrow 0$, yields:

$$
\lim _{\alpha \rightarrow 0} \frac{v_{k}\left(x_{k}+\alpha\left(x_{j}-x_{k}\right)\right)-v_{k}\left(x_{k}\right)}{\alpha} \leq \lim _{\alpha \rightarrow 0} \frac{v_{j}\left(x_{j}\right)-v_{j}\left(x_{j}-\alpha\left(x_{j}-x_{k}\right)\right)}{\alpha} .
$$

By strict monotonicity of utilities, $v_{j}$ and $v_{k}$ are differentiable almost everywhere. Let $v_{j}$ and $v_{k}$ be differentiable at $x_{j}$ and at $x_{k}$ respectively. Then, the above inequality implies that:

$$
\nabla v_{k}\left(x_{k}\right) \cdot\left(x_{j}-x_{k}\right) \geq \nabla v_{j}\left(x_{j}\right) \cdot\left(x_{j}-x_{k}\right) .
$$

Eq. (3) holds almost everywhere for each $x_{j} \in X$ such that $x_{j} R_{0} x_{k}$ and the reverse inequality holds almost everywhere for each $x_{k} \in X$ such that $x_{k} R_{0} x_{j}$. Thus, for 
each $x \in X$ at which $v_{j}$ and $v_{k}$ are differentiable, $\nabla v_{k}(x)=\nabla v_{j}(x)$ and $v_{k}$ is identical to $v_{j}$ up to an additive constant. Since each type of preferences is shared by at least two individuals, (3) implies that, for each $i \in N, v_{i}$ is concave.

The next lemma establishes interpersonal comparability.

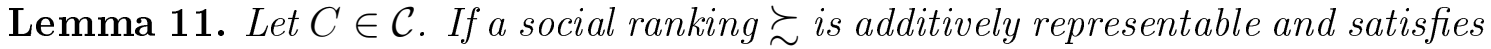
$C$-anonymous opportunities, then there exists a real-valued and monotonic function $\chi$ and constants $\left\{c_{i}\right\}_{i \in N} \in \mathbb{R}^{n}$ such that, for each $i \in N$ and each $x \in X, v_{i}(x)=$ $\chi \circ U_{i}^{C}(x)+c_{i}$.

Proof. Let $x, x^{\prime} \in X^{n}, \pi \in \Pi$, and $\left\{\lambda_{i}\right\}_{i \in N}$ be such that for some pair $j, k \in N$ :

(i) $x_{j} \in m_{j}\left(C\left(\lambda_{j}\right)\right), x_{k} \in m_{k}\left(C\left(\lambda_{k}\right)\right)$,

$x_{j}^{\prime} \in m_{j}\left(C\left(\lambda_{k}\right)\right), x_{k}^{\prime} \in m_{k}\left(C\left(\lambda_{j}\right)\right) ;$ and

(ii) $x_{i}=x_{i}^{\prime} \in m_{i}\left(C\left(\lambda_{i}\right)\right)$ for each $i \in I \backslash\{j, k\}$.

By $C$-anonymous opportunities, $x \sim x^{\prime}$. By additive representability of $\succsim$, this implies:

$$
v_{j}\left(x_{j}\right)+v_{k}\left(x_{k}\right)=v_{j}\left(x_{j}^{\prime}\right)+v_{k}\left(x_{k}^{\prime}\right) .
$$

The functions $v_{j}$ and $v_{k}$ represent preferences $R_{j}$ and $R_{k}$ respectively. Thus, there exist real-valued and monotonic functions $\chi_{j}$ and $\chi_{k}$ such that $v_{j}(x)=\chi_{j} \circ U_{j}^{C}(x)$ and $v_{k}(x)=\chi_{k} \circ U_{k}^{C}(x)$. By definition of opportunity-equivalent well-being functions, $U_{j}^{C}\left(x_{j}\right)=U_{k}^{C}\left(x_{k}^{\prime}\right)=\lambda_{j}$ and $U_{j}^{C}\left(x_{j}^{\prime}\right)=U_{k}^{C}\left(x_{k}\right)=\lambda_{k}$. Since (4) holds for each pair $x, x^{\prime} \in X^{n}$ satisfying (i) and (ii), $\chi_{j} \circ U_{j}^{C}(x)=\chi_{k} \circ U_{k}^{C}(x)+c_{k}$ for some additive constant $c_{k}$. Since this is true for each pair of individuals, the result holds.

The last step combines the previous ones and concludes the proof of Lemma 4 and, by Lemma 3, also that of Theorem 1.

Lemma 12. Lemmas 6, 10, and 11 imply Lemma 4.

Proof. By Lemmas 6 and 10, $\succsim$ can be represented by the sum of functions $v_{i}$, where for each $i \in N, v_{i}$ is a concave representation of preferences $R_{i}$. By Lemma 11, for each $i \in N$ and each $x \in X, v_{i}(x)=\chi \circ U_{i}^{C}(x)+c_{i}$. Thus, $\chi$ is such that for each $i \in N, \chi \circ U_{i}^{C}(x)$ is concave or, equivalently, $\chi \in \Psi^{C}$. By Lemma $1, \chi$ can be decomposed in a least joint concave transformation $\phi \in \Phi^{C}$ and a realvalued and concave function $f$. Since the additive constants are ordinally irrelevant, $W(x)=\sum_{i \in N} f \circ \phi \circ U_{i}^{C}\left(x_{i}\right)$ represents $\succsim$ for some real valued and concave function $f$ and any $\phi \in \Phi^{C}$. 


\section{Proof of Theorem 3}

Proof. Condition (a) implies condition $(\bar{a})$, while condition (b) is identical to condition $(\bar{b})$. It follows that $\mathcal{R}^{*} \subset \overline{\mathcal{R}}$ and (i) is satisfied. By Theorem 1, also (ii) holds.

We show next that for each $R \in \overline{\mathcal{R}} \backslash \mathcal{R}$, no social ranking $\succsim$ satisfies the axioms. Let $R \in \overline{\mathcal{R}} \backslash \mathcal{R}$. Then, there exists an individual $i \in N$ whose preferences $R_{i}$ do not admit a concave representation. By weak Pareto, continuity, separability, and possibility of trade-offs, for each $j \in N$ there exists a numerical representation of preferences $R_{j}$, say $v_{j}: X \rightarrow \mathbb{R}$, such that for each pair $x, x^{\prime} \in X^{n}, x \succsim x^{\prime} \Leftrightarrow$ $\sum_{j \in N} v_{j}\left(x_{j}\right) \geq \sum_{j \in N} v_{j}\left(x_{j}^{\prime}\right)$ (see Lemma 6). By equal-preference transfer, for each $j \in N, v_{j}$ needs to be a concave representation of preferences (see Lemma 10). This is a contradiction.

\section{References}

Aaberge, R. and Colombino, U. (2013). Using a microeconometric model of household labour supply to design optimal income taxes*, The Scandinavian Journal of Economics 115(2): 449-475.

Arrow, K. J. (1963). Social choice and individual values, 2nd. Ed., Wiley, New York and New Haven.

Arrow, K. J. (1967). Public and private values, Human values and economic Policy pp. 221.

Atkinson, A. B. (1970). On the measurement of inequality, Journal of economic theory 2(3): 244-263.

Atkinson, A. B. (2003). Multidimensional deprivation: contrasting social welfare and counting approaches, Journal of Economic Inequality 1(1): 51-65.

Atkinson, A. B. (2011). The restoration of welfare economics, The American Economic Review pp. 157-161.

Atkinson, A. B. and Bourguignon, F. (1982). The comparison of multi-dimensioned distributions of economic status, Review of Economic Studies 49(2): 183-201.

Barbera, S., Sonnenschein, H. and Zhou, L. (1991). Voting by committees, Econometrica 59: 595-609.

Bargain, O., Decoster, A., Dolls, M., Neumann, D., Peichl, A. and Siegloch, S. (2013). Welfare, labor supply and heterogeneous preferences: evidence for europe and the us, Social Choice and Welfare 41(4): 789-817.

Bergson, A. (1938). A reformulation of certain aspects of welfare economics, Quarterly Journal of Economics 52(2): 310-334. 
Blackorby, C. and Donaldson, D. (1988). Money metric utility: A harmless normalization?, Journal of Economic Theory 46(1): 120-129.

Bosmans, K., Decancq, K. and Ooghe, E. (2015). What do normative indices of multidimensional inequality really measure?, Journal of Public Economic . forthcoming.

Ching, S. and Serizawa, S. (1998). A maximal domain for the existence of strategy-proof rules, Journal of Economic Theory 78(1): 157-166.

Dalton, H. (1920). The measurement of the inequality of incomes, Economic Journal 30(119): 348-361.

d'Aspremont, C. (1985). Axioms for social welfare orderings, in D. S. L. Hurwicz and H. Sonnenschein (eds), Social Goals and Social Organizations: Essays in Memory of Elisha Pazner, Cambridge University Press, Cambridge, pp. 19-76.

d'Aspremont, C. and Gevers, L. (1977). Equity and the informational basis of collective choice, Review of Economic Studies 44: 199-209.

d'Aspremont, C. and Gevers, L. (2002). Social welfare functionals and interpersonal comparability, Handbook of social choice and welfare 1: 459-541.

Debreu, G. (1976). Least concave utility functions, Journal of Mathematical Economics $\mathbf{3}(2): 121-129$.

Dubois, P., Griffith, R. and Nevo, A. (2014). Do prices and attributes explain international differences in food purchases?, American Economic Review 104(3): 832-867.

Dworkin, R. (1981). What is equality? part 2: Equality of resources, Philosophy $\& 3$ Public Affairs pp. 283-345.

Fleming, M. (1952). A cardinal concept of welfare, Quarterly Journal of Economics pp. 366-384.

Fleurbaey, M. (2009). Beyond gdp: The quest for a measure of social welfare, Journal of Economic Literature pp. 1029-1075.

Fleurbaey, M. and Maniquet, F. (2005). Fair social orderings when agents have unequal production skills, Social Choice and Welfare 24(1): 93-127.

Fleurbaey, M. and Maniquet, F. (2006). Fair income tax, Rev Econ Stud 73(1): 55-83.

Fleurbaey, M. and Maniquet, F. (2011). A Theory of Fairness and Social Welfare, Cambridge University Press.

Fleurbaey, M. and Maniquet, F. (2014). Fairness and well-being measurement, mimeo.

Fleurbaey, M. and Tadenuma, K. (2014). Universal social orderings: An integrated theory of policy evaluation, inter-society comparisons, and interpersonal comparisons*, The Review of Economic Studies 81: 1071-1101.

Fleurbaey, M. and Trannoy, A. (2003). The impossibility of a paretian egalitarian, Social Choice and Welfare 21(2): 243-263.

Gorman, W. M. (1968). The structure of utility functions, The Review of Economic Studies 35: $367-390$.

Halevy, Y., Persitz, D., Zrill, L. et al. (2015). Parametric recoverability of preferences, Technical report, Working paper. 
Hammond, P. J. (1991). Interpersonal comparisons of utility: Why and how they are and should be made, in J. Elster and J. Roemer (eds), Interpersonal comparisons of well-being, Cambridge University Press London, pp. 200-254.

Heathcote, J., Storesletten, K. and Violante, G. L. (2014). Consumption and labor supply with partial insurance: An analytical framework, The American Economic Review 104(7): 2075-2126.

Heckman, J. J. (2001). Micro data, heterogeneity, and the evaluation of public policy: Nobel lecture, Journal of political Economy 109(4): 673-748.

Kannai, Y. (1977). Concavifiability and constructions of concave utility functions, Journal of Mathematical Economics 4(1): 1-56.

Kannai, Y. (2004). When is individual demand concavifiable?, Journal of Mathematical Economics 40(1): 59-69.

Kemp, M. C. and Ng, Y.-K. (1976). On the existence of social welfare functions, social orderings and social decision functions, Economica 43: 59-66.

Kihlstrom, R. E. and Mirman, L. J. (1981). Constant, increasing and decreasing risk aversion with many commodities, Review of Economic Studies 48: 271-280.

Kirman, A. P. (1992). Whom or what does the representative individual represent?, The Journal of Economic Perspectives pp. 117-136.

Mandler, M. (2006). Cardinality versus ordinality: A suggested compromise, American Economic Review 96(4): 1114-1136.

Maniquet, F. and Sprumont, Y. (2004). Fair production and allocation of an excludable nonrival good, Econometrica 72(2): 627-640.

Maniquet, F. and Sprumont, Y. (2005). Welfare egalitarianism in non-rival environments, Journal of Economic Theory 120(2): 155-174.

Mas-Colell, A. (1985). The Theory of General Economic Equilibrium: A Differentiable Approach., Cambridge University Press, Cambridge.

Maskin, E. (1978). A theorem on utilitarianism, Review of Economic Studies 45: 93-96.

Mayston, D. J. (1974). The idea of social choice, Macmillan London.

Mayston, D. J. (1982). The generation of a social welfare function under ordinal preferences, Mathematical Social Sciences 3(2): 109-129.

McKenzie, L. (1957). Demand theory without a utility index, The Review of Economic Studies 24: 185-189.

Ooghe, E., Schokkaert, E. and Van de gaer, D. (2007). Equality of opportunity versus equality of opportunity sets, Social Choice and Welfare 28(2): 209-230.

Parks, R. P. (1976). An impossibility theorem for fixed preferences: a dictatorial bergsonsamuelson welfare function, Review of Economic Studies 43: 447-450.

Pazner, E. (1979). Aggregation and Revelation of Preferences, Elsevier, North-Holland, Amsterdam and New York, chapter Equity, Nonfeasible Alternatives and Social Choice: A Reconsideration of the Concept of Social Welfare, pp. 161-173.

Pigou, A. C. (1912). Wealth and Welfare, Macmillan, London. 
Rawls, J. (1971). A Theory of Justice, Harvard University Press.

Saez, E. and Stantcheva, S. (2015). Generalized social marginal welfare weights for optimal tax theory, American Economics Review . forthcoming.

Samuelson, P. A. (1947). Fundations of Economic Analysis, Harvard University Press, Cambridge.

Samuelson, P. A. (1977). Reaffirming the existence of "reasonable" bergson-samuelson social welfare functions, Economica 44: 81-88.

Samuelson, P. A. and Swamy, S. (1974). Invariant economic index numbers and canonical duality: Survey and synthesis, American Economic Review 64: 566-593.

Sen, A. (1979). Equality of what?, Tanner Lecture on human values.

Sen, A. (1992). Inequality reexamined, Oxford University Press.

Slesnick, D. T. (1998). Empirical approaches to the measurement of welfare, Journal of Economic Literature pp. 2108-2165.

Suppes, P. (1966). Some formal models of grading principles, Synthese 16: 284-306.

Thomson, W. (1994). Notions of equal, or equivalent, opportunities, Social Choice and Welfare 11(2): 137-156. 